Annales Geophysicae (2003) 21:761-777 (C) European Geosciences Union 2003

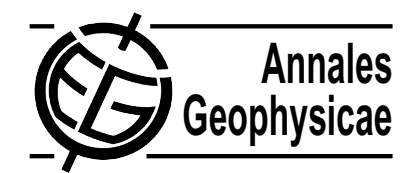

\title{
Multi-frequency observations of E-region HF radar aurora
}

\author{
S. E. Milan ${ }^{1}$, M. Lester ${ }^{1}$, and N. Sato ${ }^{2}$ \\ ${ }^{1}$ Department of Physics and Astronomy, University of Leicester, Leicester LE1 7RH, UK \\ ${ }^{2}$ National Institute of Polar Research, Tokyo 173-8515, Japan
}

Received: 18 July 2002 - Revised: 21 October 2002 - Accepted: 23 October 2002

\begin{abstract}
Multi-frequency observations of E-region coherent backscatter from decametre waves reveal that auroral echoes tend to comprise two spectral components superimposed, one at low Doppler shifts, below $250 \mathrm{~m} \mathrm{~s}^{-1}$, and the other Doppler shifted to near the ion-acoustic speed or above, up to $800 \mathrm{~m} \mathrm{~s}^{-1}$. The low Doppler shift component occurs at all look directions; Doppler shifts near the ion acoustic speed occur when looking at low flow angles along the direction of the electron drift in the electrojet, and Doppler shifts in excess of the ion acoustic speed occur at intermediate flow angles. The latter population appears most commonly at radar frequencies near $10-12 \mathrm{MHz}$, with its occurrence decreasing dramatically at higher frequencies. The velocity of the high Doppler shift echoes increases with increasing radar frequency, or irregularity wave number $k$. The velocity of the low Doppler shift population appears to be suppressed significantly below the line-of-sight component of the electron drift. Initial estimates of the altitude from which scatter occurs suggest that the high Doppler shift echoes originate from higher in the E-region than the low Doppler shift echoes, certainly in the eastward electrojet. We discuss these observations with reference to the theories of de/stabilization of twostream waves by electron density gradients and electrostatic ion cyclotron waves excited by field-parallel electron drifts.
\end{abstract}

Key words. Ionosphere (ionospheric irregularities)

\section{Introduction}

The instability mechanisms which give rise to field-aligned irregularities in the collisional plasma of the auroral E-region ionosphere have been the subject of much study over the last several decades. Other than in situ rocket measurements, the most powerful technique for investigating the nature of the irregularities is by determining the Doppler spectra of radio waves coherently scattered from the plasma density perturbations. The frequency of the probing radio wave determines

Correspondence to: S. E. Milan (Steve.Milan@ion.le.ac.uk) the irregularity scale-length from which scatter arises, with radars in the HF, VHF, and UHF bands being sensitive to scale-lengths of tens of metres, metres, and fractions of metres, respectively. Thus, the wavelength- or $k$-dependence of the instability mechanisms can be investigated by employing a range of radar frequencies.

Most work has been conducted with VHF radars. Early in these studies it was recognized that the Doppler spectra of echoes backscattered from the E-region fell into several broad categories, defined mainly by the spectral characteristics of Doppler shift and spectral width. Borrowing from earlier work conducted in the equatorial region, Balsley and Ecklund (1972) introduced the "type" nomenclature, in which there appeared two predominant classes: type I echoes, with a narrow peak Doppler-shifted to near the ion-acoustic speed, and type II spectra which are broader, with Doppler shifts below the ion-acoustic speed. These have been identified as echoes from two-stream and gradient drift waves, respectively (see, for instance, Haldoupis, 1989; Sahr and Fejer, 1996; and references therein). Few echoes are observed by VHF radars with Doppler shifts in excess of the expected ambient E-region ion-acoustic speed (near $400 \mathrm{~m} \mathrm{~s}^{-1}$ ), and this was thought for some time to be an upper limit for the irregularity drift speed, irrespective of the background electron drift which is controlled by the convection electric field and can reach several $\mathrm{km} \mathrm{s}^{-1}$. However, some work has suggested that the ion-acoustic speed is not such a limiting factor after all, e.g. Keys and Johnston (1979) in the VHF band, and Foster and Erickson (2000) at UHF. There is some debate as to whether such echoes are scattered from new irregularity types, for instance, electrostatic ion cyclotron waves, or arise in regions where the ion-acoustic speed is elevated due to heating (raising the threshold velocity); one major obstacle to answering such questions is that experiments rarely measure the ion-acoustic speed within the scattering volume, and thus its value must be inferred. Overall, the "type" nomenclature is perhaps now seen as too restrictive and poorly-defined to allow the physics behind the generation of irregularities to be properly elucidated, and sta- 
tistical approaches to classifying spectral populations can be more useful (e.g. Watermann et al., 1989).

More recent work involving HF radars has demonstrated significant similarities and also dissimilarities between the echo characteristics observed at metre and decametre wavelengths (e.g. Villain et al., 1987, 1990; Hanuise et al., 1991; Milan and Lester, 1999, 2001; Milan et al., 2001, 2002). Chief among the dissimilarities is the frequent observation at HF of echoes with Doppler shifts considerably enhanced above the ambient ion-acoustic speed. These differences suggest a considerable wavelength or $k$ dependence in the Eregion irregularity characteristics. Some attempts have been made to compare echo characteristics at widely differing frequencies, for instance, in the HF and VHF bands (e.g. Koustov et al., 2002). In this study, however, we concentrate on a narrower $k$-range, employing the frequency-agility ( 8 to $20 \mathrm{MHz}$ ) of the SuperDARN radars (Greenwald et al., 1995) to investigate the characteristics of echoes from irregularities with wavelengths in the range 8 to $19 \mathrm{~m}$. Key to this investigation is the new "Stereo" capability of the Iceland East SuperDARN radar, which allows measurement of backscatter echo spectra at two radar frequencies simultaneously.

\section{The Stereo CUTLASS experiment}

The standard SuperDARN HF coherent scatter radar experimental arrangement and the "myopic" scan mode have been described in some detail before (Greenwald et al., 1995 and Milan and Lester, 2001, respectively), and only a brief summary will be given here. The radars are frequency-agile between 8 and $20 \mathrm{MHz}$, though for transmission license considerations they are constrained to operate within a few allocated frequency bands within this range, each typically a few hundred $\mathrm{kHz}$ in width. The finite width of the bands allows automatic selection of an interference-free frequency prior to each radar measurement. The radars sound along 16 beam directions, separated by $\sim 3.2^{\circ}$ of azimuth. In normal operation each beam is integrated for 3 or $7 \mathrm{~s}$, resulting in scanning periods of 1 or $2 \mathrm{~min}$, and is gated into 75 range cells of $45 \mathrm{~km}$ in length, with a range to the first cell of $180 \mathrm{~km}$. The radars transmit a 7-pulse multi-pulse scheme, which yields 17-lag autocorrelation functions, from which power, Doppler velocity, and spectral width can be determined (Hanuise et al., 1993). In addition, interferometric measurements made by a pair of antenna arrays allows the elevation angle of the return echoes to be measured, which, in turn, gives a very approximate estimate of the altitude from which the echoes originate (Milan et al., 1997, 2001; Milan and Lester, 2001). Without a detailed knowledge of the electron density profile of the ionosphere, the level of refraction experienced by the radar radio wave is unknown (though it is assumed that the ray is refracted to orthogonality with the magnetic field, such that backscatter can be observed). This limits the accuracy of the altitude estimates, as straight-line propagation must be assumed. This results generally in an overestimate of the scattering altitude, though the error is thought

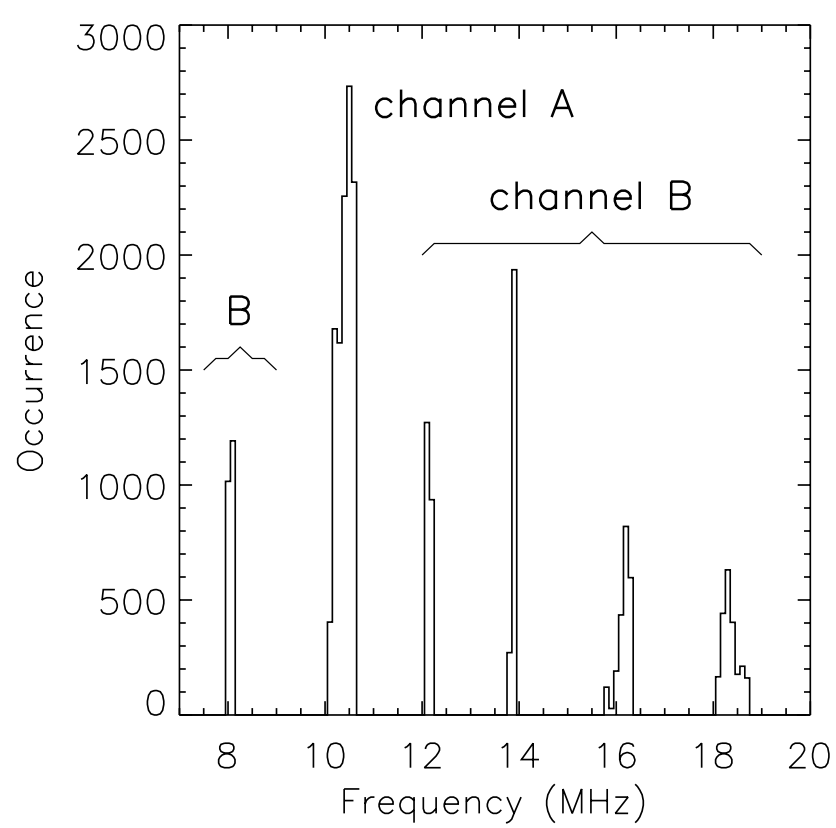

Fig. 1. Occurrence of transmission at different operating frequencies by the Iceland East radar during 18:00-06:00 UT, 14-15 December 2001. Channel A of the Stereo system transmitted near $10 \mathrm{MHz}$, whereas the transmissions of channel B were split between five frequency bands near $8,12,14,16$, and $18 \mathrm{MHz}$.

to be less than $10-20 \mathrm{~km}$ in the E-region (see also Milan et al., 2001). The Iceland East radar, one of the CUTLASS radars (see Fig. 12) which form the eastern-most pair of the Northern Hemisphere SuperDARN chain, has been used extensively in the past for the study of E-region backscatter. As part of these studies the "myopic" mode was developed in which the range separation is decreased to $15 \mathrm{~km}$, to give high spatial resolution observations of the near-range fieldof-view, that portion of the field-of-view in which E-region echoes are routinely detected. Usually the myopic mode has been run with a radar operating frequency of $10 \mathrm{MHz}$ and a beam dwell period of $3 \mathrm{~s}$. The Iceland East radar, located at Pykkvibaer, Iceland $\left(63.77^{\circ} \mathrm{N}, 339.46^{\circ} \mathrm{E}\right)$, is again employed in the present study.

Recently, the versatility of the CUTLASS radars has been enhanced by the "Stereo" upgrade, which effectively turns each radar into two. In normal SuperDARN operation only a fraction of the available transmitter duty-cycle $(\sim 7 \%)$ is used to generate the radar pulse sequence. Stereo exploits some of the remaining unused duty-cycle by interleaving two pulse sequences at different radar frequencies. Then, employing the original plus an additional receiver system, it is possible to sound simultaneously on two radar "channels", known as $\mathrm{A}$ and $\mathrm{B}$. The beams of the two channels can be steered independently, and in principle, the range separation or range to the first cell of each channel can also differ. Although the two channels must be separated in frequency, the minimum separation is only $15 \mathrm{kHz}$, much less than the width of a typical SuperDARN frequency-band, allowing observations on both channels within the same band if desired. Routine op- 


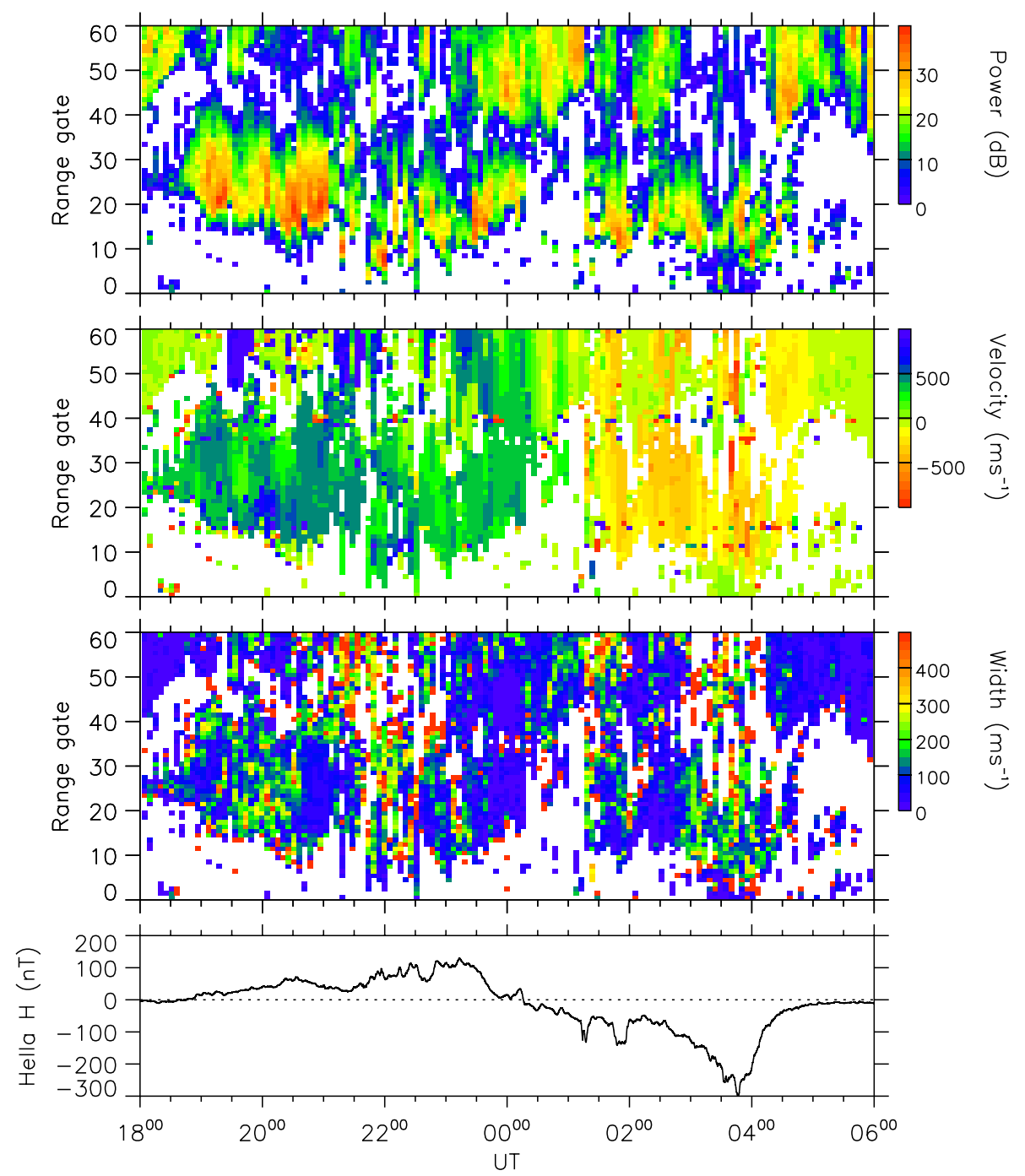

Fig. 2. (Top panels) Range-time-parameter plots of backscatter power, Doppler shift, and spectral width, from beam 15 , the most easterlypointing beam of the radar. Positive Doppler shifts indicate motion towards the radar. (Bottom panel) $H$-component magnetogram from the SAMNET Hella magnetometer, co-located with the Iceland East radar.

eration of the Stereo capability of CUTLASS commenced in January 2002, though test runs were conducted in the latter half of 2001. In December 2001 the myopic mode exploited Stereo to make multi-frequency observations of the E-region, and it is the Stereo myopic run on the night of the 14-15 December, 18:00-06:00 UT, that is the focus of the present study. Channel A operated in myopic mode as normal, at a radar frequency of $10 \mathrm{MHz}$, completing a scan of the field-of-view every $63 \mathrm{~s}$. Channel B sounded each beam simultaneously, though at a different frequency; each scan of channel B was conducted at one of 5 frequencies, in the order $8,12,14,16$, and $18 \mathrm{MHz}$, with a complete cycle being completed every $315 \mathrm{~s}$. In this way, truly simultaneous and common-volume measurements of E-region echoes at different frequencies were made, with $10 \mathrm{MHz}$ being the reference frequency. Figure 1 shows a histogram of the frequencies sounded during the 12-h experimental interval, in $100-\mathrm{kHz}$ wide bins. Division into the 6 frequency bands is clearly ev- ident, though some bands are wider than others (the 10 and $18 \mathrm{MHz}$ bands especially). Five times as many observations were made at $10 \mathrm{MHz}$ on channel $\mathrm{A}$ as in each of the channel $\mathrm{B}$ frequency bands. It is the comparison of the echo characteristics in each of these bands to which we now turn.

\section{Observations}

\subsection{Overview}

E-region backscatter is observed throughout the night of 14 15 December 2001. Figure 2 shows backscatter power, lineof-sight Doppler velocity and spectral width measurements from channel A (10 MHz) along beam 15, the most eastward directed beam of the Pykkvibaer radar, for the $12 \mathrm{~h}$ of observation. Measurements were made at this frequency with a sampling interval of $\sim 1 \mathrm{~min}$, though they are shown here for illustrative purposes at a time resolution of $\sim 5 \mathrm{~min}$, equiva- 

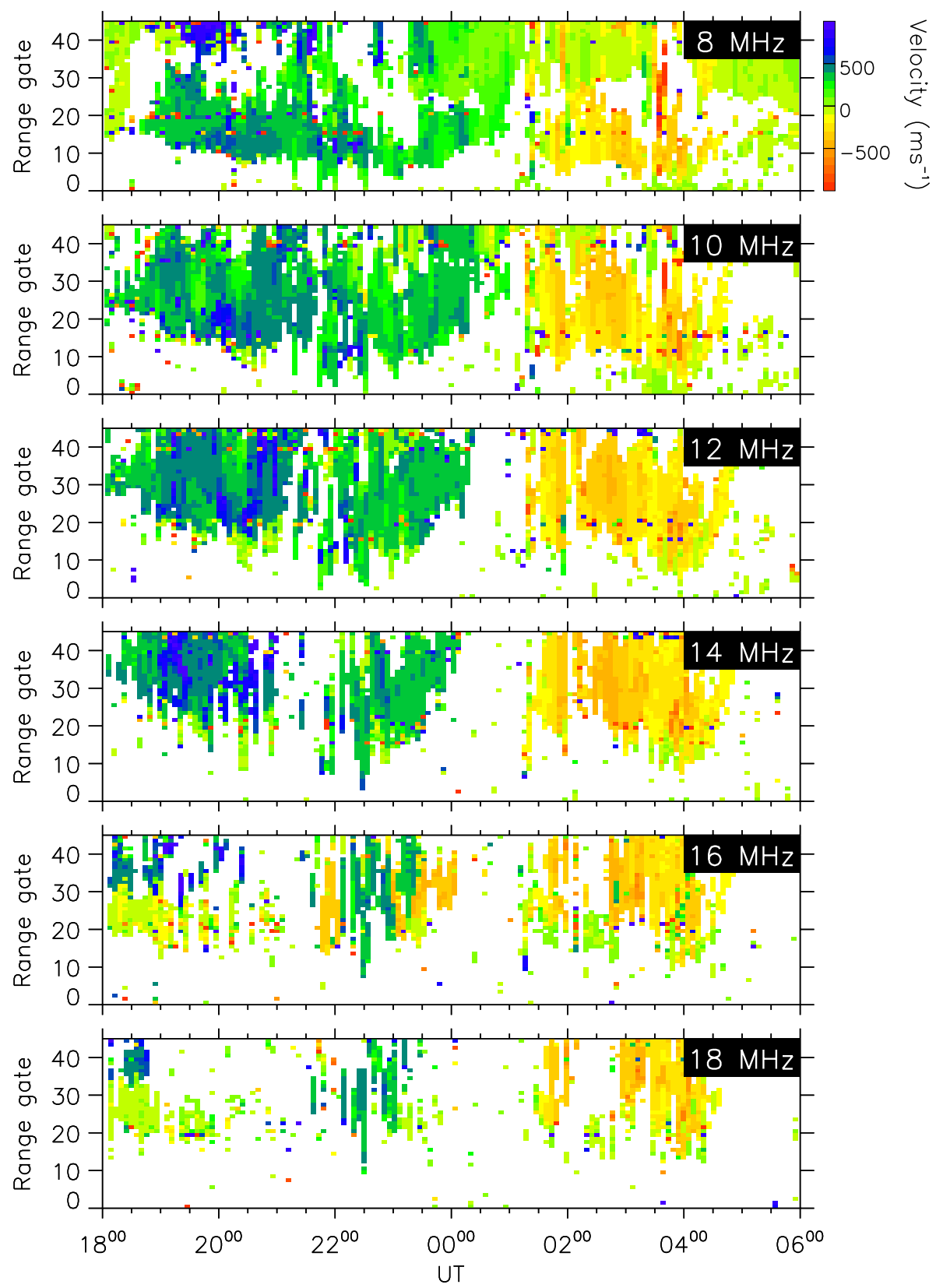

Fig. 3. Range-time-Doppler shift plots from the six frequency bands employed in the study.

lent to the resolution of measurements made in each of the five frequency bands on channel B. From the power observations it is clear that the backscatter falls into two distinct regions at nearer and further ranges, a demarcation appearing near range gate $40(\sim 800 \mathrm{~km})$. Interferometric estimates of the altitude of origin of each echo allow us to attribute backscatter at nearer ranges to the E-region, and from further ranges to the F-region. Hanuise et al. (1991) also divided their data set along similar lines, using a demarcation range of $\sim 700 \mathrm{~km}$. In the rest of this study we are interested only in backscatter from the E-region, and take great care to exclude echoes from the F-region.

As beam 15 points almost eastward, the line-of-sight velocity represents mainly the zonal component of the elec- tron drift, with positive (negative) velocities corresponding to flow towards (away from) the radar, that is westward (eastward). Prior to 01:00 UT, the measured velocity is westward, corresponding to the eastward electrojet. After this, eastward flow, corresponding to the westward electrojet, is seen. The bottom panel of Fig. 2 shows the $H$ component magnetogram from the Hella SAMNET station, co-located with the Pykkvibaer radar. The positive and negative excursions of $H$ before and after 00:00 UT are consistent with the magnetic deflections expected from the eastward and westward electrojets, respectively.

We now turn to a comparison of the backscatter observed in the six frequency bands. Figure 3 shows range-timevelocity plots from beam 15 for each of the frequency bands, 
from $8 \mathrm{MHz}$ at the top to $18 \mathrm{MHz}$ at the bottom. In general similar observations are made at each frequency, though some differences are noteworthy. (1) At $8 \mathrm{MHz}$ F-region echoes are observed at near ranges, as close as gate 20 after 23:00 UT. (2) At higher frequencies, the occurrence of E-region echoes decreases, especially above $14 \mathrm{MHz}$. (3) In addition, the minimum range at which E-region echoes are observed increases from near gate 5 at $8 \mathrm{MHz}$ to gate 20 at $18 \mathrm{MHz}$. All three of these observations are thought to arise from the frequency dependence of refraction of HF radio waves in the ionosphere.

In a given electron density profile, refraction decreases with increasing frequency. As a consequence, at higher frequencies radio waves must propagate to greater distances before being refracted to orthogonality with the magnetic field, the condition necessary for observation of backscatter from field-aligned irregularities. Conversely, at lower frequencies backscatter from either the E- or F-region can be observed at nearer ranges.

\subsection{Echo characteristics}

To examine the Doppler shift characteristics of the scatter, Fig. 4 shows histograms of the occurrence of different lineof-sight velocities observed within the E-region scatter in all beams of the radar, in the six frequency bands. Positive and negative velocities primarily comprise echoes from the eastward and westward electrojets, respectively, as discussed above. For guidance, vertical dotted lines at $\pm 400 \mathrm{~m} \mathrm{~s}^{-1}$ show approximate values of the ion-acoustic speed $C_{S}$ in the E-region. The distributions show a main central peak close to $0 \mathrm{~m} \mathrm{~s}^{-1}$, with wings extending to approximately $\pm 800 \mathrm{~m} \mathrm{~s}^{-1}$, with less than $0.5 \%$ of the observations having $|v|>800 \mathrm{~m} \mathrm{~s}^{-1}$. Within the eastward electrojet observations (positive velocities) there is a secondary peak close to $v=C_{S}$, taken in the past as evidence for the presence of echoes from two-stream waves (type I spectra) at either VHF frequencies (e.g. Haldoupis, 1989) or HF frequencies (e.g. Hanuise et al., 1991; Milan and Lester, 2001). In the westward electrojet there is less evidence for such a secondary peak, though there is clear suggestion of a shoulder in the distribution near $v=C_{S}$. Examining the occurrence distributions from individual beams of the radar we find that the low-velocity peak is observed mainly in beams which look across the direction of electrojet flow, and the high-velocity peak is observed predominantly in beams which look along the electrojets. However, in all beams there is a contribution from both high and low velocity populations.

Figure 5a shows the temporal evolution of the velocity occurrence in 5-min intervals, with $10 \mathrm{MHz}$ being selected as representative of all the frequency bands. The colourcoding indicates echo occurrence in velocity bins $50 \mathrm{~m} \mathrm{~s}^{-1}$ wide. The time-integrated occurrence distribution would be that shown in Fig. 4. The purpose of this figure is to show that, especially in the eastward electrojet, two distinct peaks in the velocity distribution are observed at most times; that is a peak near $v=0 \mathrm{~m} \mathrm{~s}^{-1}$, and a second peak near $v=C_{S}$.
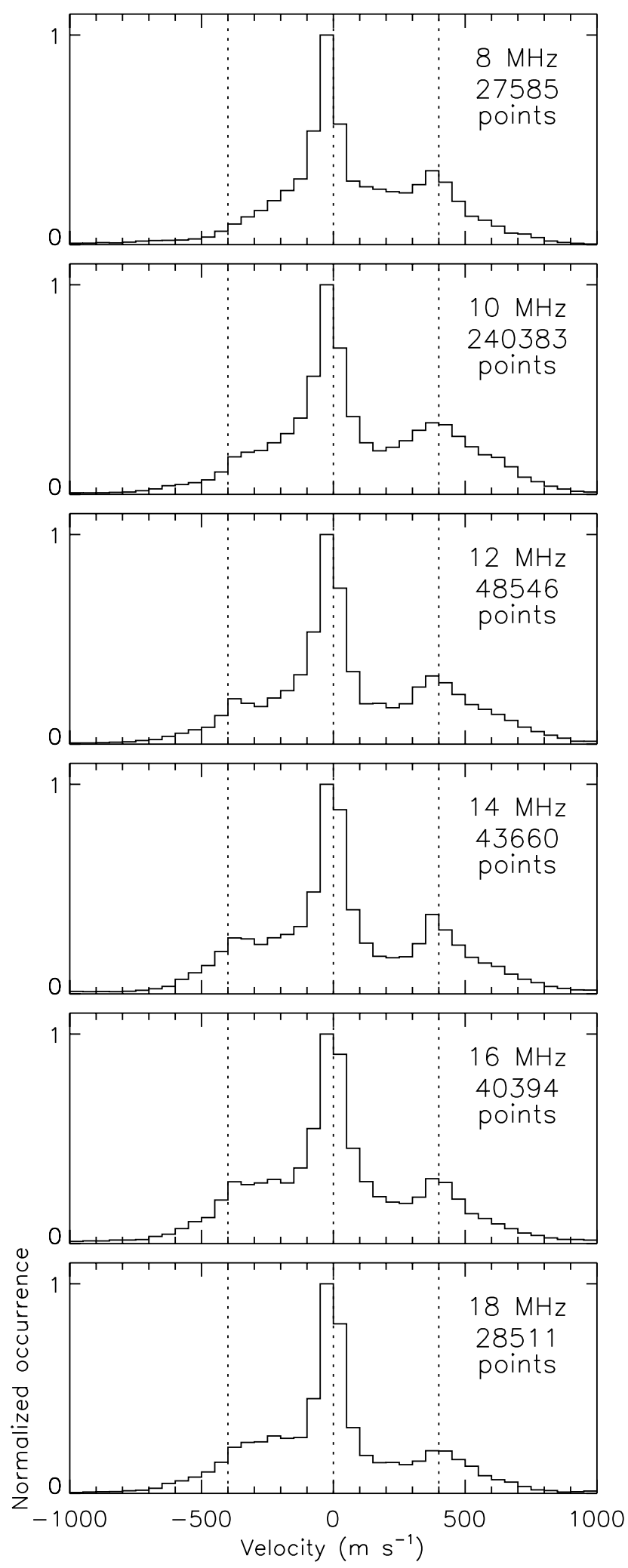

Fig. 4. Occurrence distributions of Doppler shift observed in the six frequency bands. Vertical dotted lines at $\pm 400 \mathrm{~m} \mathrm{~s}^{-1}$ approximately indicate the ion-acoustic speed. 


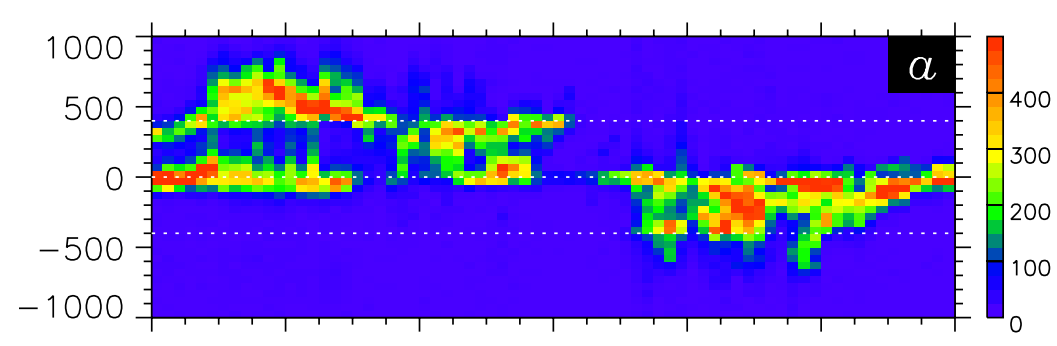

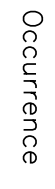

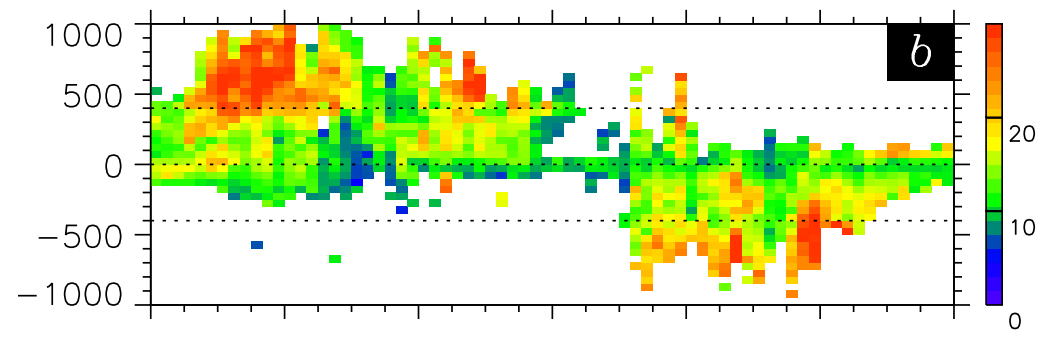

3
$\frac{3}{0}$
0
0
3
0
0
0
0
0
$\frac{0}{2}$
⿹
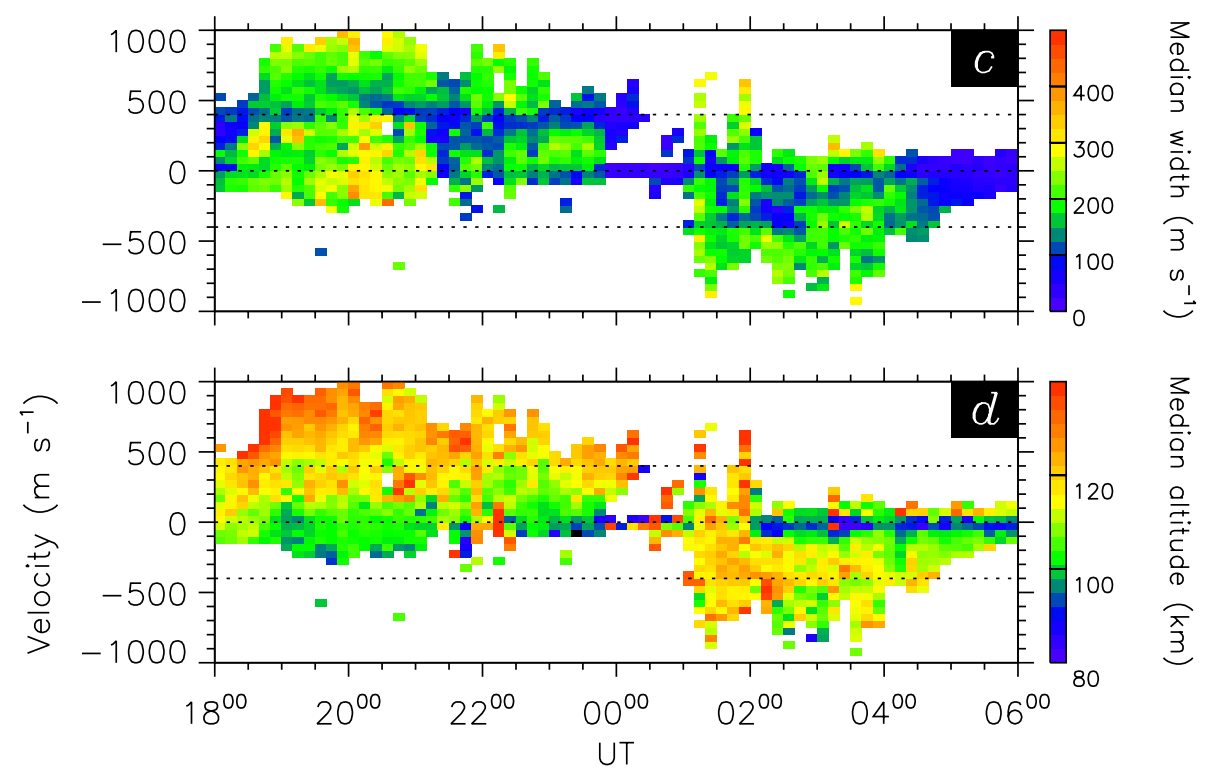

Fig. 5. (a) Five-minute occurrence distributions of Doppler shift from Channel A. (b-d) Median values of power, spectral width, and estimated altitude of scatter, respectively, where significant numbers of echoes are recorded.

Both peaks in the distribution are quite narrow at any instant in time, though variations in the position of the high velocity peak results in this being somewhat smeared out in the integrated distribution. In general, when one population appears the other is present also, and this is particularly apparent in the higher frequency bands where echoes are seen more sporadically (see Fig. 3), i.e. high and low Doppler shift echoes tend to occur together, and we refer to these as our high and low Doppler shift populations.

Figures $5 b$ and $c$ show the spectral characteristics of the echoes. These figures indicate, in each time and velocity bin where significant numbers of echoes (more than 10) are observed, the median echo power (Fig. 5b) and median spectral width (Fig. 5c). In addition, Fig. 5d indicates the median altitude from which the echoes are received. Several populations of echoes are observed in these panels. After 00:00 UT, echoes of very low Doppler velocity (less than $50 \mathrm{~m} \mathrm{~s}^{-1}$ ), very low width (less than $100 \mathrm{~m} \mathrm{~s}^{-1}$ ) and low signal-to-noise ratio $(10 \mathrm{~dB})$ are seen to originate from very low altitudes in the E-region, perhaps below $90 \mathrm{~km}$. These we attribute to echoes from meteor trails (Hall et al., 1997), which are not of direct interest to this study. The incidence of meteor scatter is greatest in the morning local time sector due to the orbital motion of the Earth. Prior to 00:00 UT, in the eastward electrojet, low velocity echoes tend to be of higher backscatter power $(15 \mathrm{~dB})$, are broader $\left(200 \mathrm{~m} \mathrm{~s}^{-1}\right.$ and more) and originate at higher altitudes, 100 to $110 \mathrm{~km}$. Such echoes are also present in the westward electrojet, but they are somewhat outnumbered by the meteor echoes. These broad, low Doppler shift echoes have similar characteristics to type II spectra observed with VHF radars. This, then, is the low Doppler shift population.

At higher velocities, approaching the ion-acoustic speed near $400 \mathrm{~m} \mathrm{~s}^{-1}$, the altitude from which echoes are received 

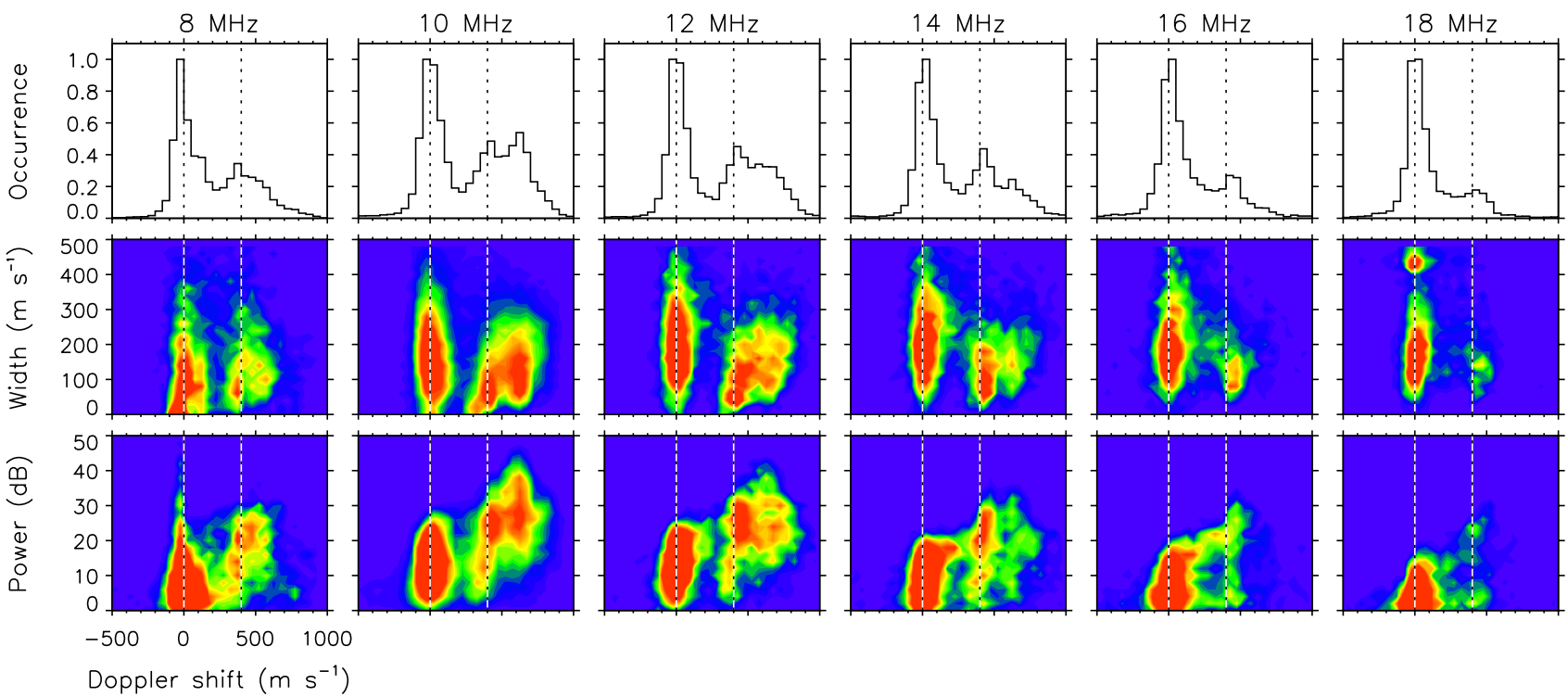

Fig. 6. Echo characteristics in the six frequency bands sounded, for the interval 18:00 UT to 20:00 UT. (Top panels) Occurrence distributions of Doppler shift. (Middle panels) Occurrence distributions of spectral width versus Doppler shift. (Bottom panels) Occurrence distributions of backscatter power versus Doppler shift. In these distributions, red indicates greatest occurrence.

increases. Close to the ion-acoustic speed the median width of echoes is low again $\left(150 \mathrm{~m} \mathrm{~s}^{-1}\right.$ and less), especially in the eastward electrojet. Low spectral widths at Doppler shifts near the ion-acoustic speed are characteristic of type I spectra. At higher velocities again, above the ion-acoustic speed, the altitude from which echoes are received increases, the spectra become broader once more, and the backscatter power increases markedly. We class these echoes, with velocities near the ion-acoustic speed and above, as our high Doppler shift population, though we will further subdivide this into two categories, $C_{S}$ and super- $C_{S}$ echoes, as explained below. We note that the echo characteristics described here are fully consistent with those reported at greater length by Milan and Lester (2001).

To reiterate this last point, Fig. 6 presents a more detailed analysis of the echo types observed in the scatter. Here we concentrate on a limited portion of the observations, 18:00 to 20:00 UT, to avoid the smearing issues described above. For each frequency band the top panels indicate the selfnormalized Doppler shift occurrence, similar to that shown in Fig. 4. Below these are occurrence distributions of echo Doppler shift versus spectral width, sometimes known as Watermann plots (Watermann et al., 1989). In these plots red indicates the greatest occurrence. Below these are similar distributions of Doppler shift versus power. In all these distributions, a clear demarcation between the low and high Doppler shift population is found near $250 \mathrm{~m} \mathrm{~s}^{-1}$. In addition, it is seen that the high Doppler shift population can be further subdivided into two, with peaks near 400 and $650 \mathrm{~m} \mathrm{~s}^{-1}$, especially in the 10,12 , and $14 \mathrm{MHz}$ frequency bands. As $400 \mathrm{~m} \mathrm{~s}^{-1}$ is close to the nominal ion-acoustic speed, we will refer to these two populations as the " $C_{S}$ " and "super- $C_{S}$ " echoes in the rest of the paper. Above $14 \mathrm{MHz}$ the super- $C_{S}$ population becomes much less evident.

The low Doppler shift population has the broadest range of spectral widths, from near $0 \mathrm{~m} \mathrm{~s}^{-1}$ up to $500 \mathrm{~m} \mathrm{~s}^{-1}$. The backscatter power in this population tends to be less than $25 \mathrm{~dB}$, and decreases with increasing radar frequency. The $C_{S}$ population has the lowest spectral widths, in general below $200 \mathrm{~m} \mathrm{~s}^{-1}$, and powers up to $25 \mathrm{~dB}$. Peak spectral widths in the super- $C_{S}$ population reach $350 \mathrm{~m} \mathrm{~s}^{-1}$. These echoes also display the greatest backscatter power, varying between 15 and $50 \mathrm{~dB}$ at $10 \mathrm{MHz}$, though decreasing with increasing frequency. The echo characteristics described here, especially at $10 \mathrm{MHz}$, are just as reported by Milan and Lester (2001) and shown in their Figs. 8 and 9. Here, however, we are able to reveal the frequency dependence of the power and occurrence of the differing echo types. We separate the observations into three Doppler shift regimes corresponding to the low, $C_{S}$, and super- $C_{S}$ populations: $|v|<250 \mathrm{~m} \mathrm{~s}^{-1}, 250<v<550 \mathrm{~m} \mathrm{~s}^{-1}$, and $v>550 \mathrm{~m} \mathrm{~s}^{-1}$. Figure 7 presents the frequency dependence of the relative occurrence of echoes and the median power for each of these populations, where the occurrence in the $10 \mathrm{MHz}$ frequency band has been divided by 5 , to account for sampling differences. The low Doppler shift echoes are the most populous, with only a slight decrease in occurrence with increasing frequency, down $75 \%$ over the frequency range. About half as common are the $C_{S}$ echoes, though their occurrence drops to $35 \%$ of their peak as frequency increases. At 10 and $12 \mathrm{MHz}$ super- $C_{S}$ are almost as common as $C_{S}$ echoes. However, their occurrence is greatly reduced in the other frequency bands, and they are almost nonexistent at $18 \mathrm{MHz}$. The median powers of all three populations increase between 8 and $10 \mathrm{MHz}$, after which there is a tendency for power to decrease with increasing frequency. The depressed pow- 

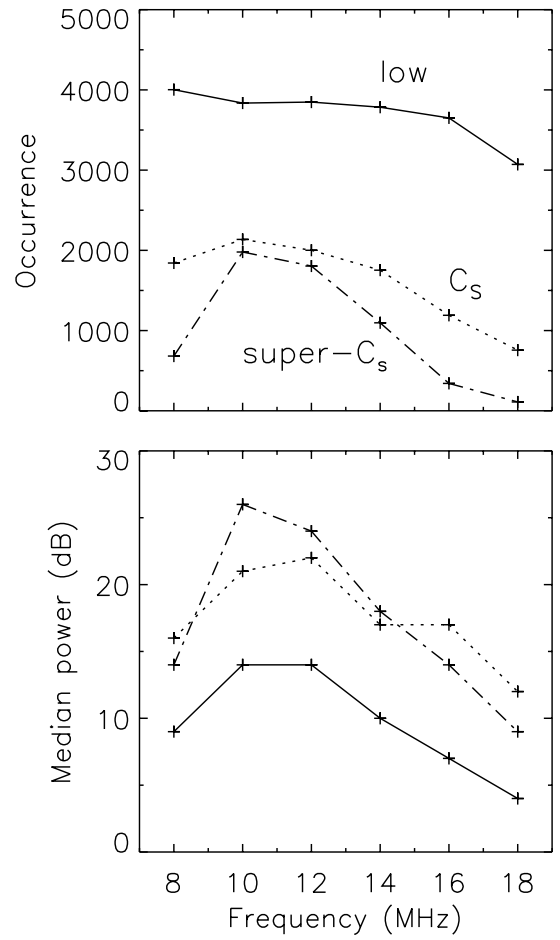

Fig. 7. Frequency variation of occurrence (top panel) and median power (bottom panel) of the low Doppler shift, $C_{S}$ and super- $C_{S}$ echo populations.

ers at $8 \mathrm{MHz}$ are probably related to the high absorption of the radar signals in the D-region, which falls as the inverse square of the radar frequency. On the other hand, the large decrease in the super- $C_{S}$ echo occurrence and power at $8 \mathrm{MHz}$ suggests perhaps that this down-turn is also due to a decrease in backscatter cross section of this population. The variation of power with frequency of the low Doppler shift population is similar to that of the $C_{S}$ echoes, though the latter are greater by approximately $8 \mathrm{~dB}$. Between 10 and $14 \mathrm{MHz}$, the super- $C_{S}$ echoes are of the greatest power of all three populations, though the decrease with frequency is the steepest of all. Overall, the occurrence and power variation of the super- $C_{S}$ population would appear to explain the dearth of such echoes at VHF frequencies.

\subsection{Two-component echoes (TWOPEEs)}

We now turn to a direct comparison of Doppler shifts observed at different frequencies. By comparing simultaneous and co-located measurements of echo characteristics in the two stereo channels, we can form occurrence distributions of the Doppler shift at $10 \mathrm{MHz}$ compared with the Doppler shift in the other frequency bands. These distributions are shown in Fig. 8, one panel for each frequency band sounded by channel B. The colour-coding shows the density of points within the distribution plane, with red representing the greatest density. To aid discussion, regions of the distribution plane are labeled in the bottom right-hand corner of Fig. 8 . Several populations of points are seen in the distributions.
The majority of points reveal a close association between velocities measured in the two channels, that is fall in regions 1 and 2 of the distributions. However, a significant proportion of the points fall in regions 3 and 4 of the distribution, that is where a high Doppler shift is measured on channel B and a low Doppler shift on channel A (region 3) or vice versa (region 4). That these points form distinct populations in regions 3 and 4 mirrors the distinction between high and low Doppler shift echoes found in Fig. 4. Although these populations are most apparent in the eastward electrojet (positive velocities), they are present in the westward electrojet as well. In Fig. 8 we have superimposed horizontal and vertical lines to draw a distinction between high and low Doppler shifts, employing a threshold of $200 \mathrm{~m} \mathrm{~s}^{-1}$. We now make a further distinction between regions 1 and 2: although these points show a rough equality of velocity, region 1 represents high Doppler shift echoes measured on both channel A and $\mathrm{B}$, and region 2 represents low Doppler shifts measured on the two channels.

To demonstrate more clearly how the two channels can observe high and low Doppler shift echoes simultaneously in the same location, Fig. 9 presents backscatter observations from two scans starting at 18:44:57 UT and 18:55:25 UT, when channel A was sounding at $10 \mathrm{MHz}$ and channel $\mathrm{B}$ at $14 \mathrm{MHz}$. Lines which curve from the bottom-left to top-right represent lines of geomagnetic latitude from $66^{\circ}$ to $74^{\circ}$ in steps of $2^{\circ}$ and lines which curve from top-left to bottomright represent lines of geomagnetic longitude in steps of $5^{\circ}$. E-region backscatter features are those which occur below a latitude of $70^{\circ}$. The echo regions curve from bottom-left to top-right, indicating that they are roughly L-shell aligned, as discussed previously by Milan and Lester (2001). In both scans, both channels A and B observe backscatter in the same locations, though the Doppler shift characteristics depend on frequency. During the first scan indicated, both channel A and B observed a very similar pattern of line-of-sight velocities in the lower portion of the E-region backscatter, that is between approximately $66^{\circ}$ and $68^{\circ}$ latitude. However, poleward of this, between $68^{\circ}$ and $70^{\circ}$ latitude, considerable differences are observed: at $10 \mathrm{MHz}$ on channel A Doppler velocities below $200 \mathrm{~m} \mathrm{~s}^{-1}$ are measured, whereas on channel $\mathrm{B}$ at $14 \mathrm{MHz}$ Doppler velocities in excess of $400 \mathrm{~m} \mathrm{~s}^{-1}$ are seen. In other words, there are two distinct regions within the backscatter, in one of which observations are broadly similar at the two frequencies, and in the other of which the low Doppler shift population is observed at $10 \mathrm{MHz}$ and the high Doppler shift population at $14 \mathrm{MHz}$. Close examination of the observations indicates that there are also regions where low Doppler shifts are seen on channel B and high Doppler shifts on channel A. Figure 10 shows the comparison of Doppler shifts observed in the two channels from this scan, in a similar format to Fig. 8. The grouping of points in the regions described above is clear. In the second example shown in Fig. 9, the pattern and mix of Doppler shifts in the two channels are more complicated. However, Fig. 10 demonstrates that exactly the same relationship between high and low Doppler shift populations exists. 

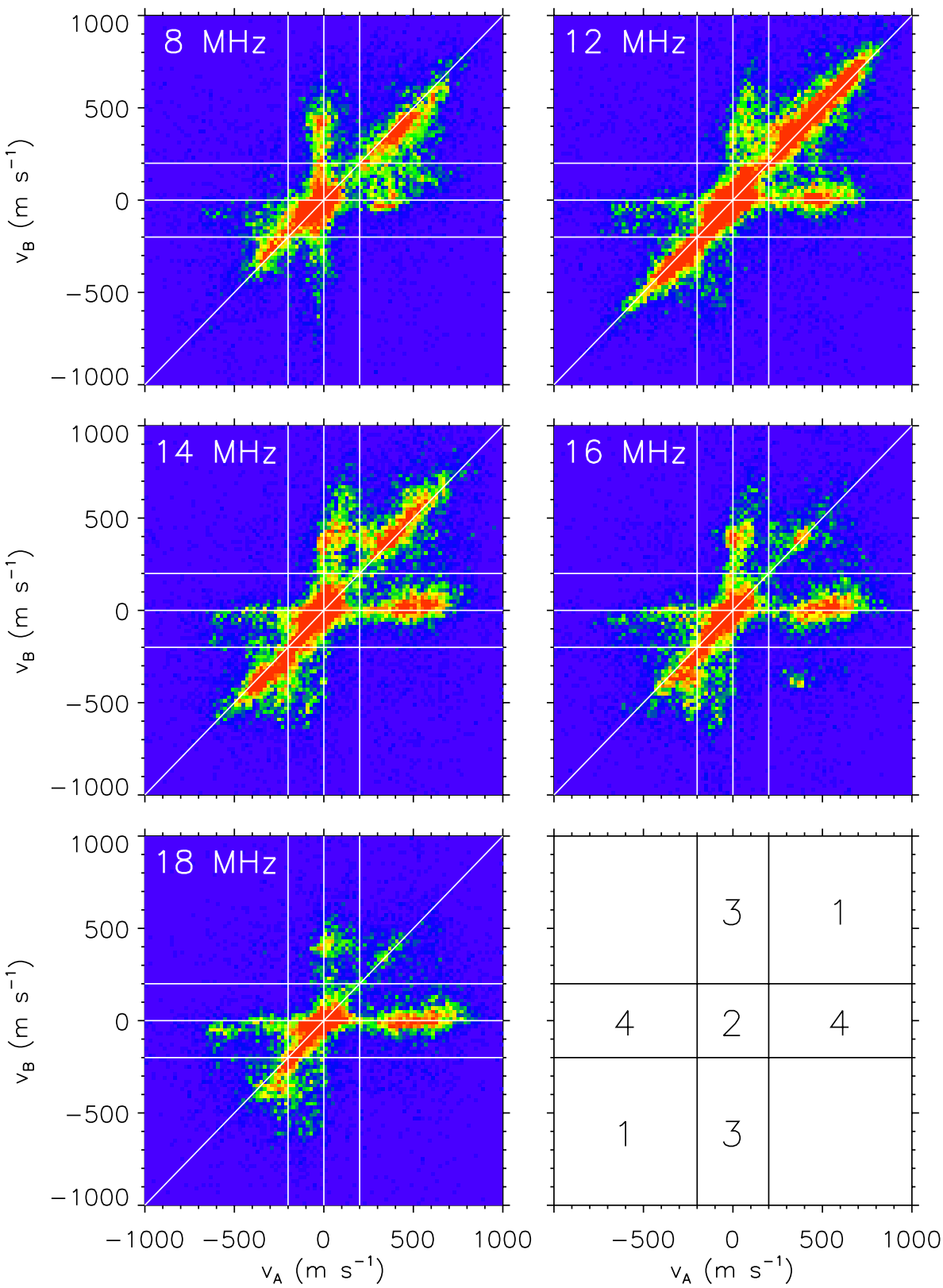

Fig. 8. Occurrence distributions showing the relationship between Doppler shift measured on channel $\mathrm{A}\left(v_{A}\right)$ at $10 \mathrm{MHz}$ with Doppler shift measured on channel $\mathrm{B}\left(v_{B}\right)$ in each of the five channel B frequency bands. Red indicates greatest occurrence. The bottom right-hand panel numbers different regions of the distribution plane to aid discussion.

Our observations suggest that throughout the electrojet regions the E-region ionosphere is stratified into two altitude regimes. Low Doppler shift echoes are scattered from the lower stratum, and high Doppler shift echoes from the upper stratum. Different radar frequencies can pick out scatter from one or the other of the strata, depending on the altitude at which the orthogonality condition is met, which, in turn, depends upon the level of refraction in the ionosphere. This raises the possibility that, if the orthogonality condition is met over a range of altitudes, then high and low Doppler shift components should be observed in the same echo spectrum, and this is indeed found to be the case. Figure 11 shows the echo spectra from the first scan, 18:44:57 UT, of Fig. 9. Here we concentrate solely on range gates 25 to 32 , that portion of the field-of-view in which some of the most significant differences between channels A and B are observed. The spectra are shown self-normalized, in the Doppler shift range $1000 \mathrm{~m} \mathrm{~s}^{-1}$, vertical lines indicating $0 \mathrm{~m} \mathrm{~s}^{-1}$. Examination of the channel A spectra indicate that they comprise, in general, only a single main peak. To the left of the field-ofview, they display a small Doppler shift, though to the right the Doppler shift approaches the ion-acoustic speed, $C_{S}$. In contrast, the channel B spectra to the left of the field-of-view tend to show two components, one at low and the other at 

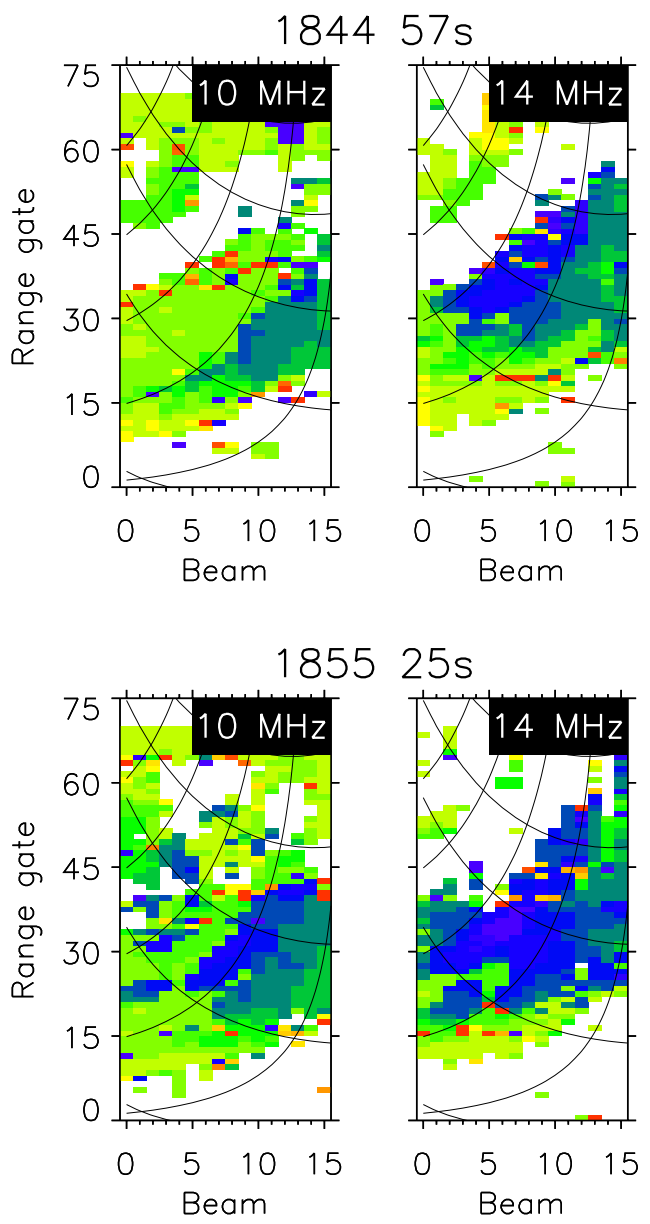

Fig. 9. (Top panels) Doppler shift measurements on channel A and B (10 and $14 \mathrm{MHz}$, respectively) from the scan starting 18:44:57 UT. Superimposed curves show constant geomagnetic longitude (top-left to bottom-right) and latitude (top-right to bottomleft). Latitudes are $66^{\circ}, 68^{\circ}, 70^{\circ}, 72^{\circ}$ and $74^{\circ}$. (Bottom panels) Similar to above, though for the scan starting 18:55:25 UT.

high Doppler shifts. To the right of the field-of-view, predominantly single-peaked, high Doppler shift spectra are observed similar to channel A, though in some radar cells there is again evidence for a low Doppler shift component.

Thus, even at a single frequency, two components can be observed in each spectrum, indicating that backscatter is observed from a range of altitudes. The relative intensity of the two components will depend on the altitudes at which the orthogonality condition is met (keeping in mind that scatter may also occur from slightly off-orthogonal irregularities), on the backscatter cross section of the irregularities at each altitude, and on the amount of focusing the radar beam has experienced at each altitude. The first and last of these will depend greatly on radar frequency and propagation path, leading to the variations observed between channels in Fig. 9 . Comparing Figs. 9 and 11 it is clear that when two peaks are present in the echo spectra the SuperDARN ACF analysis technique picks out the velocity of the component with the highest power. The spectral width parameter that is de-

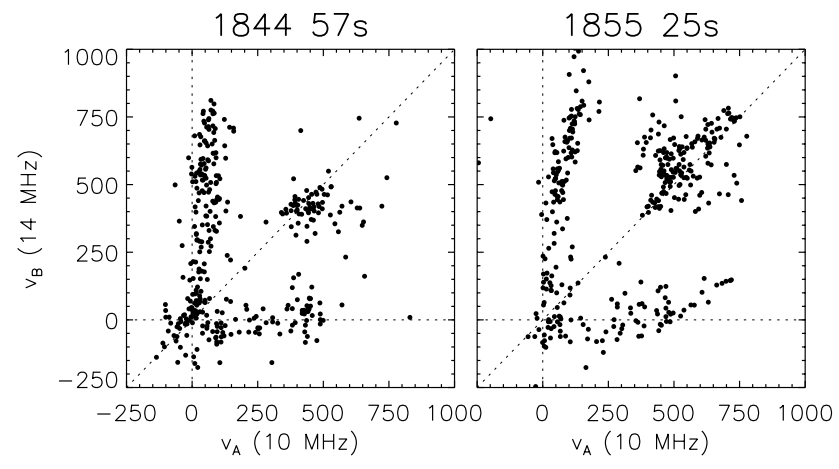

Fig. 10. Similar to Fig. 8, showing a comparison of Doppler shifts measured on channels A and B for the two scans presented in Fig. 9.

duced, however, tends to be related to the separation of the peaks, and not of the width of the main peak. Thus, great care must be taken when interpreting HF radar spectral width in the context of instability mechanisms. Re-examination of other myopic campaign observations indicates that such two-component spectra are a common and characteristic feature of E-region echoes, and for this reason we give them the name "two-peak E-region echoes" or TWOPEEs.

Returning to Fig. 10 (and also Fig. 8), there is some suggestion in the region 3 and 4 populations that there exists a relationship between $v_{A}$ and $v_{B}$. This is not surprising, as the instability mechanisms giving rise to the irregularities responsible for both the high and low Doppler shift echoes will be in part driven by the background electric field, which is independent of altitude. To a first approximation, in region 3: $v_{A} \approx 0.1 v_{B}$; in region 4: $v_{B} \approx 0.1 v_{A}$. However, the two regions are not exactly symmetrical, and in region 4 it appears that $v_{B}$ can be negative when $v_{A}$ is positive, and vice versa in region 3. This implies that there may be an angular offset in the preferred directions of irregularity phase propagation at different altitudes.

Next we investigate the flow angle characteristics of the observed Doppler shifts, that is the variation of Doppler shift with the angle between the radar look-direction and the electron drift, assuming that the electron drift in the electrojet regions is approximately zonal. In Fig. 12, for the two scans of Fig. 9, the Doppler shift is shown as a function of L-shell angle, $\phi$, the angle between the radar beam and the local Lshell at each point within the radar field-of-view. Low values of $\phi$, where the radar beams are directed along L-shells, correspond to the eastern portion of the field-of-view; in more westerly beams $\phi$ tends to higher values, i.e. the beams look across the electrojet flow. Several echo populations are found in the distributions, consistent with previous studies, and readers are directed to Milan and Lester (2001) for a preliminary discussion of such figures. In channel A, both low and high Doppler shift echoes are seen, the high Doppler shifts clustering near values of $C_{S}\left(\sim 400 \mathrm{~m} \mathrm{~s}^{-1}\right)$ and being present only at lower L-shell angles $\left(\phi<30^{\circ}\right)$. In channel B, fewer low Doppler shift echoes are observed, but a new higher Doppler shift population is present at high L-shell an- 

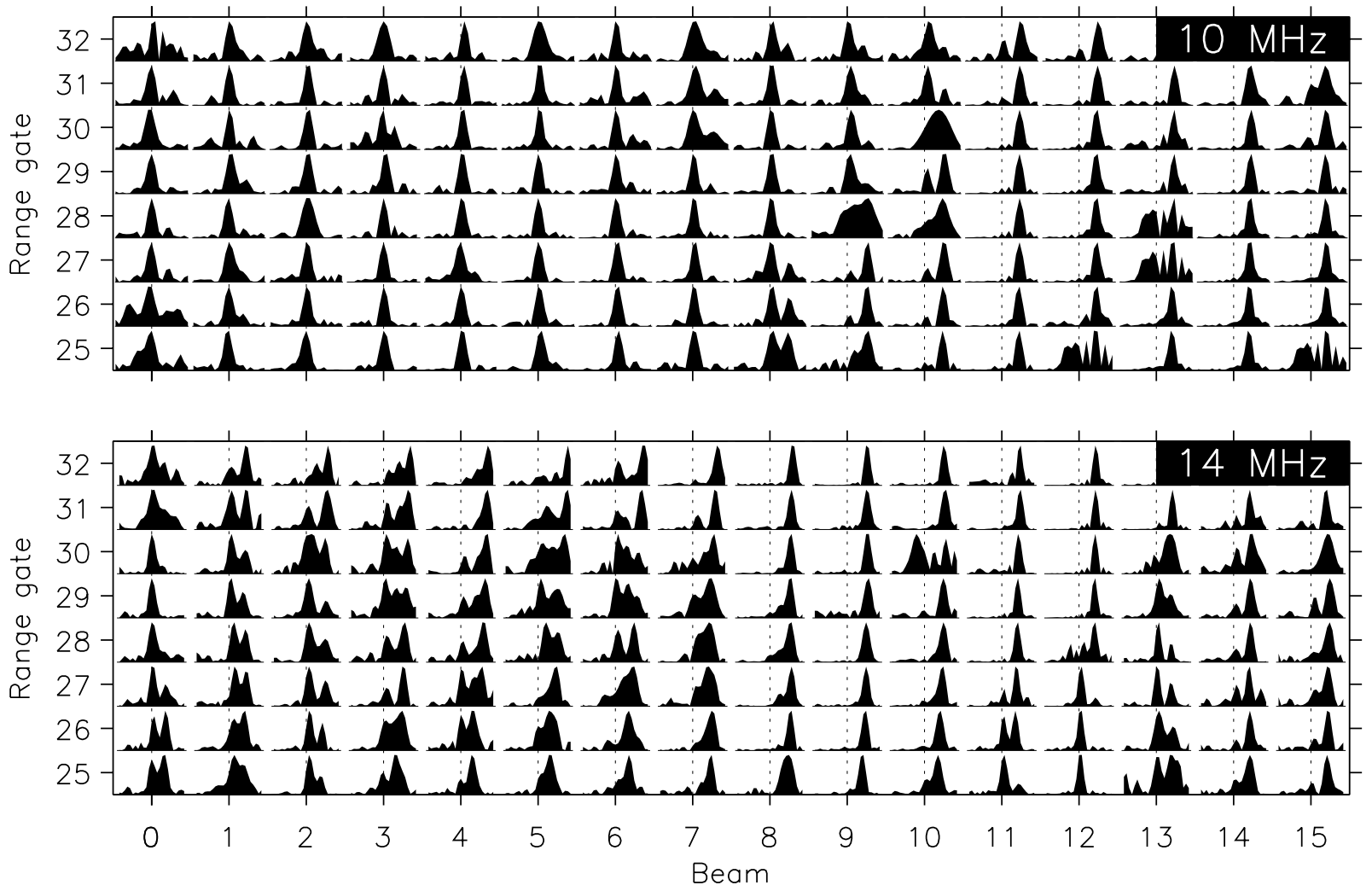

Fig. 11. Doppler spectra of echoes observed on channel A (top panel) and B (bottom panel) for the scan starting 18:44:57 UT, as shown at the top of Fig. 9. Each spectrum is shown self-normalized, in the velocity range $\pm 1000 \mathrm{~ms}^{-1}$.

gles $\left(\phi>30^{\circ}\right)$. Arrows indicate those points which have low Doppler shifts in channel A but high Doppler shifts in channel B.

As before, we can separate our high Doppler shift observations into two categories, those with Doppler shifts near the ion-acoustic speed at low flow angles and those with Doppler shifts in excess of the ion-acoustic speed at higher flow angles, the $C_{S}$ and super- $C_{S}$ populations, respectively. These latter echoes tend to be seen when looking significantly across the electrojet flow. Milan and Lester (2001) showed, as do our Figs. 5 and 6, that those echoes with Doppler shifts near $C_{S}$ have narrow spectra (type I); those with higher Doppler shifts are broader and of higher backscatter power. In addition to these echoes, the low Doppler shift population is seen across all flow angles, and tends to have broader spectra (type II).

\subsection{Frequency dependence of Doppler shift}

Finally, we turn to the frequency-dependence of the high Doppler shift echoes. Examination of Fig. 8 shows that when high Doppler shift echoes are observed in both channels A and B simultaneously (region 1 of the distributions), echoes recorded by the higher frequency channel tend to be of higher velocity. This effect is relatively small, but can be seen most clearly by comparing the 8 and $12 \mathrm{MHz}$ panels of Fig. 8 . To quantify the effect, we select all points from region 1 and form occurrence distributions of the ratio $v_{B} / v_{A}$, as shown in Fig. 13. Although the distributions are quite broad, there is a clear offset from $v_{B} / v_{A}=1$ at each frequency. Bestfit Gaussian curves are superimposed on each distribution to help identify the offset, which is shown as a function of frequency in the bottom right-hand panel. This reveals that the velocity of the high Doppler shift population tends to increase as a function of radar frequency, or in other words, with increasing irregularity $k$.

\section{Discussion}

Original studies of the spectral shapes of VHF radar backscatter echoes from the E-region found two dominant forms (e.g. Balsley and Ecklund, 1972; Haldoupis, 1989; Sahr and Fejer, 1996; and references therein): type I spectra, narrow with Doppler shifts close to the ion-acoustic speed, believed to be echoes from two-stream waves; and broader, slower type II echoes, thought to be scattered from gradient drift waves. Types III $\left(v \approx 0.5 C_{S}\right)$ and IV $\left(v>C_{S}\right)$ were also identified, but these are much rarer. More recent experiments conducted at HF frequencies (Hanuise et al., 1991; Villain et al., 1987, 1990; Milan and Lester, 1999, 2001; Milan et al., 2001, 2002) indicate that similarities and dissimilarities exist for echoes from E-region irregularities with decametre wavelengths. For instance, both type I and type II spectra can be identified, but also a significant proportion of echoes with Doppler shifts in excess of the ion-acoustic 
speed are observed. Differences are to be expected for two main reasons. First, theory predicts this. For instance, electron density gradients are expected to influence the irregularity characteristics more at decametre wavelengths than at metre wavelengths, modifying the threshold conditions for irregularity generation (e.g. Fejer et al., 1984). Second, refraction of HF radar signals in the ionosphere means that orthogonality with the magnetic field can be achieved over a range of altitudes, whereas VHF radars are constrained to almost line-of-sight geometries. If HF radars can access different heights within the E-region, then different ambient conditions and possibly instability mechanisms will be probed. Reflecting this range of possible effects, the superion-acoustic echoes identified in HF radar observations have been widely interpreted as electrostatic ion cyclotron waves in the upper E-region (Villain et al., 1987, 1990) or gradientstabilized two-stream waves (St.-Maurice et al., 1994). One way to distinguish between these interpretations is to investigate the $k$-dependence of the echo characteristics and compare with theory. An obvious method of achieving this is to conduct multi-frequency observations of E-region backscatter, which was the aim of the present experiment.

The present study confirms previous findings, that is backscatter echoes fall into three distinct populations, mainly defined by their Doppler shift: (a) a low Doppler shift population, in which the Doppler shift rarely exceeds $200 \mathrm{~m} \mathrm{~s}^{-1}$; (b) echoes with Doppler shifts close to the nominal ionacoustic speed $C_{S} \approx 400 \mathrm{~m} \mathrm{~s}^{-1}$, which we term $C_{S}$ echoes; (c) echoes with Doppler shifts in excess of the nominal ionacoustic speed, up to $800 \mathrm{~m} \mathrm{~s}^{-1}$, which we term the super- $C_{S}$ population. The first two categories were labeled populations $i i$ and $i$, respectively, in the classification scheme of Milan and Lester (2001). The super- $C_{S}$ echoes were further subdivided into populations $i i i$ and $i v$ by Milan and Lester based on their flow angle dependence, which essentially recognizes that super- $C_{S}$ echoes occur at intermediate flow angles, neither looking along the electrojet flow nor across it. We take care to state that $C_{S}$ echoes appear at the nominal ion-acoustic speed, as in fact we do not measure this. It is possible that super- $C_{S}$ echoes arise in regions where the ionacoustic speed is elevated above its usual value. However, we feel that the flow-angle dependence of the occurrence of super- $C_{S}$ echoes argues against this.

A major finding of the present study is that while low Doppler shift and $C_{S}$ echoes are observed across the frequency range probed ( 8 to $18 \mathrm{MHz}$ ), the occurrence and backscatter power of the super- $C_{S}$ echoes peaks near 10 and $12 \mathrm{MHz}$. (This frequency dependence again argues against super- $C_{S}$ echoes being associated with elevated $C_{S}$.) The obvious conclusion is that this is due to a $k$-dependence of the scatter cross section of the irregularities responsible for the super- $C_{S}$ echoes, though propagation variations (refraction, focusing, absorption, etc.) must always be kept in mind when working in the HF band. This is especially true as it appears that different echo populations originate from different altitudes within the E-region. This conclusion arises from our second major finding that high and low Doppler shift echoes can be observed at the same location at different frequencies, or indeed can be simultaneously present in a single spectrum (hence, two-peak E-region echoes or TWOPEEs). This co-location suggests that the irregularities from which these echoes scatter must be stratified. Interferometer observations in the eastward electrojet indicate that the high Doppler shift population originates at a higher altitude in the topside Eregion (Fig. 5); though in the westward electrojet the observations are less clear-cut. In the past, using radar measurements at a single frequency, Villain et al. $(1987,1990)$ and Milan et al. (2001, 2002) observed small, irregular patches of high Doppler shift echoes superimposed on a background of lower Doppler shift backscatter. Milan et al. (2001) reported interferometric measurements which suggested that the lower Doppler shifts originated from altitudes near the peak of the E-region, and higher Doppler shifts some $20 \mathrm{~km}$ higher, and showed echo spectra in which both high and low Doppler shift components were present. (To avoid confusion, Milan et al. also discussed a lower D-region population of scatterers, but these appear rare and are not of concern to the present study.) However, it was not clear from these studies that the stratified echoes co-existed over extensive regions of the electrojets. In principle, every echo received from the E-region can contain two velocity components arising from different altitudes, but that which dominates depends on the radar ray-path through the ionosphere, which in turn, depends on the operating frequency. In some regard this idea is similar to the proposal of Uspensky et al. (1994, 2001) that echo characteristics are determined by the convolution of radar propagation and irregularity characteristics integrated over a range of altitudes.

Villain et al. (1987, 1990) also observed low and high Doppler shift populations. Comparison of our Fig. 10 with Figs. 3, 4 and 6 of Villain et al. (1987) indicates very close accord in the flow angle characteristics observed: a low Doppler shift population in which velocity decreases with increasing flow angle, a population of echoes with Doppler shifts near $C_{S}$ at low flow angles, and a population of super$C_{S}$ echoes at intermediate flow angles. Milan and Lester (2001) showed that this pattern is reproduced in almost all HF radar E-region observations. Villain et al. $(1987,1990)$ attributed the low Doppler shift population to gradient drift waves, and the $C_{S}$ and super- $C_{S}$ echoes to two-stream and electrostatic ion cyclotron waves, respectively. In this interpretation, the velocity of the low Doppler shift echoes should be close to the line-of-sight component of the electron drift in the electrojet, that is $(E / B) \cos \theta$, where $\theta$ is the flow angle, which is equal to the L-shell angle $\phi$ defined above if the flow is zonal. In regions where this exceeds the ion-acoustic speed two-stream waves should be generated, in which case the line-of-sight velocity should saturate at $C_{S}$ (such regions are said to be within the "cone of instability"). However, in neither our example, nor those of Villain et al. does the velocity of the low Doppler shift echoes approach the ionacoustic speed (suggesting convection electric field of the order of $10 \mathrm{mV} \mathrm{m}^{-1}$ and less), and hence, no points within the radar field-of-view should be within the cone of instability. 
$184457 s$

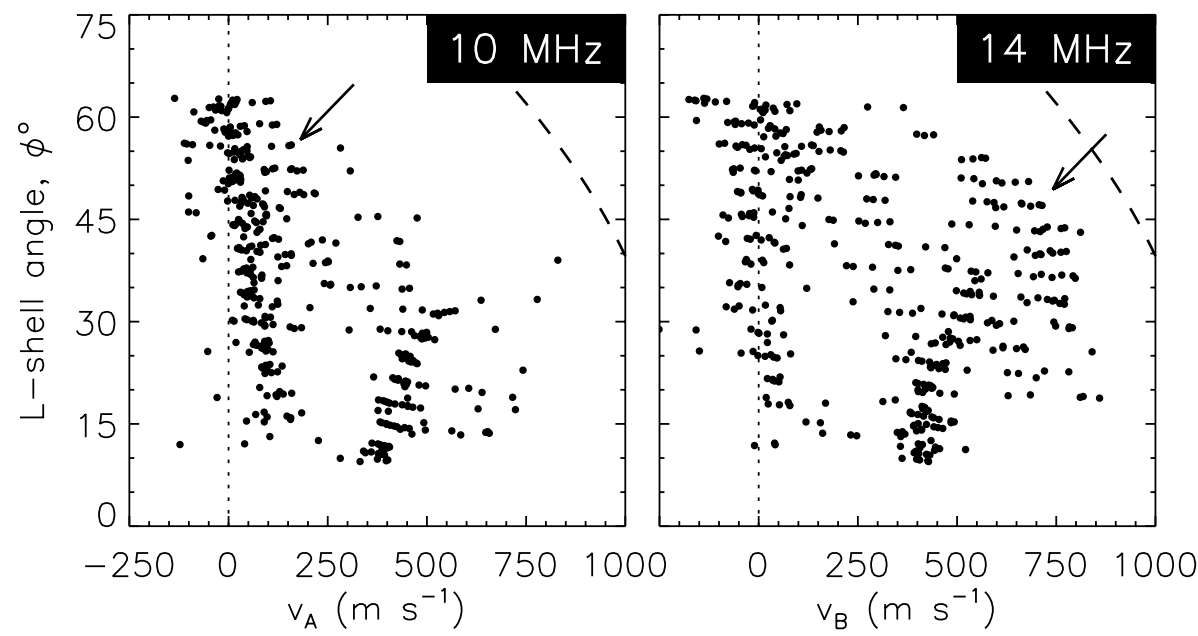

$185525 s$

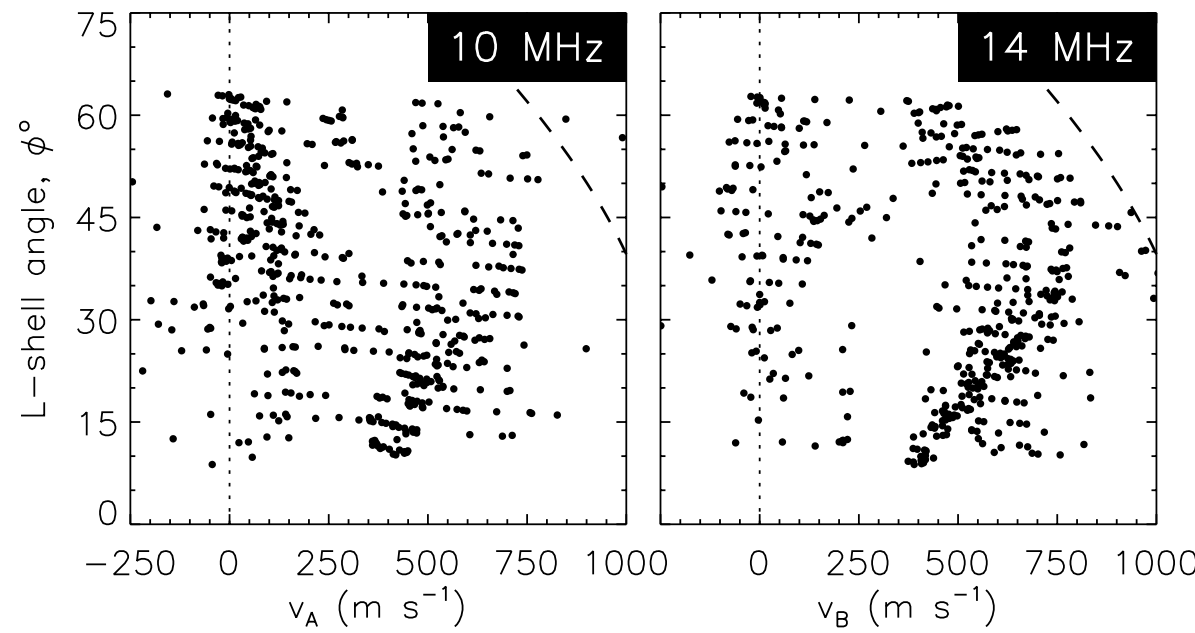

Fig. 12. Doppler shift as a function of L-shell angle, $\phi$, for the scans shown in Fig. 9. Superimposed are dashed curves indicating $v_{l o s}=$ $1100 \cos \left(\phi-15^{\circ}\right)$.

For this reason Villain et al. suggested that the two-stream and ion cyclotron waves were generated in regions of subcritical electric field, and postulated that field-aligned electron drifts must be present to drive the E-region unstable.

However, it seems doubtful to us that the electric field should always be so low during these measurements (and the much more extensive data set of Milan and Lester, 2001), as electrojet electric fields of many $10 \mathrm{~s}$ of $\mathrm{mV} \mathrm{m}^{-1}$ are common. Consequently, it seems sensible to suggest that the velocity of the low Doppler shift echoes is depressed significantly below the line-of-sight component of the electron drift. As $C_{S}$ echoes are only observed at low flow angles these may indeed be within the cone of instability. If this is the case, it is straightforward to place a lower threshold on the background electric field: looking to Fig. 12, $C_{S}$ echoes are observed for $\phi<30^{\circ}$, suggesting that $E / B \geq 400 / \cos 30^{\circ} \approx 500 \mathrm{~m} \mathrm{~s}^{-1}$, corresponding to $E \approx 25 \mathrm{mV} \mathrm{m}^{-1}$. With this electric field, however, the super- $C_{S}$ echoes still have Doppler shifts in excess of the line-of-sight component of the electron drift. Unless fieldparallel drifts are to be invoked to drive these, they also place a lower threshold on the electric field, though in this case of the order of $50 \mathrm{mV} \mathrm{m}^{-1}$.

Clearly, a knowledge of the background electric field is desirable. Unfortunately, electric field measurements colocated with the E-region backscatter are difficult to acquire due to a lack of suitable instrumentation within the Iceland East radar field-of-view. Overpassing satellites could provide such information, and this we intend to feature in a future study. For the present, however, we can estimate the electric field from measurements made nearby, by the Finland leg of the CUTLASS system. Figure 14a shows the Finland and Iceland East radar fields-of-view at 18:45 UT (see also Fig. 9) in a magnetic latitude and local time coordinate system. The Finland radar is operating with the normal range resolution of $45 \mathrm{~km}$ and a scan time of $1 \mathrm{~min}$. All backscatter 

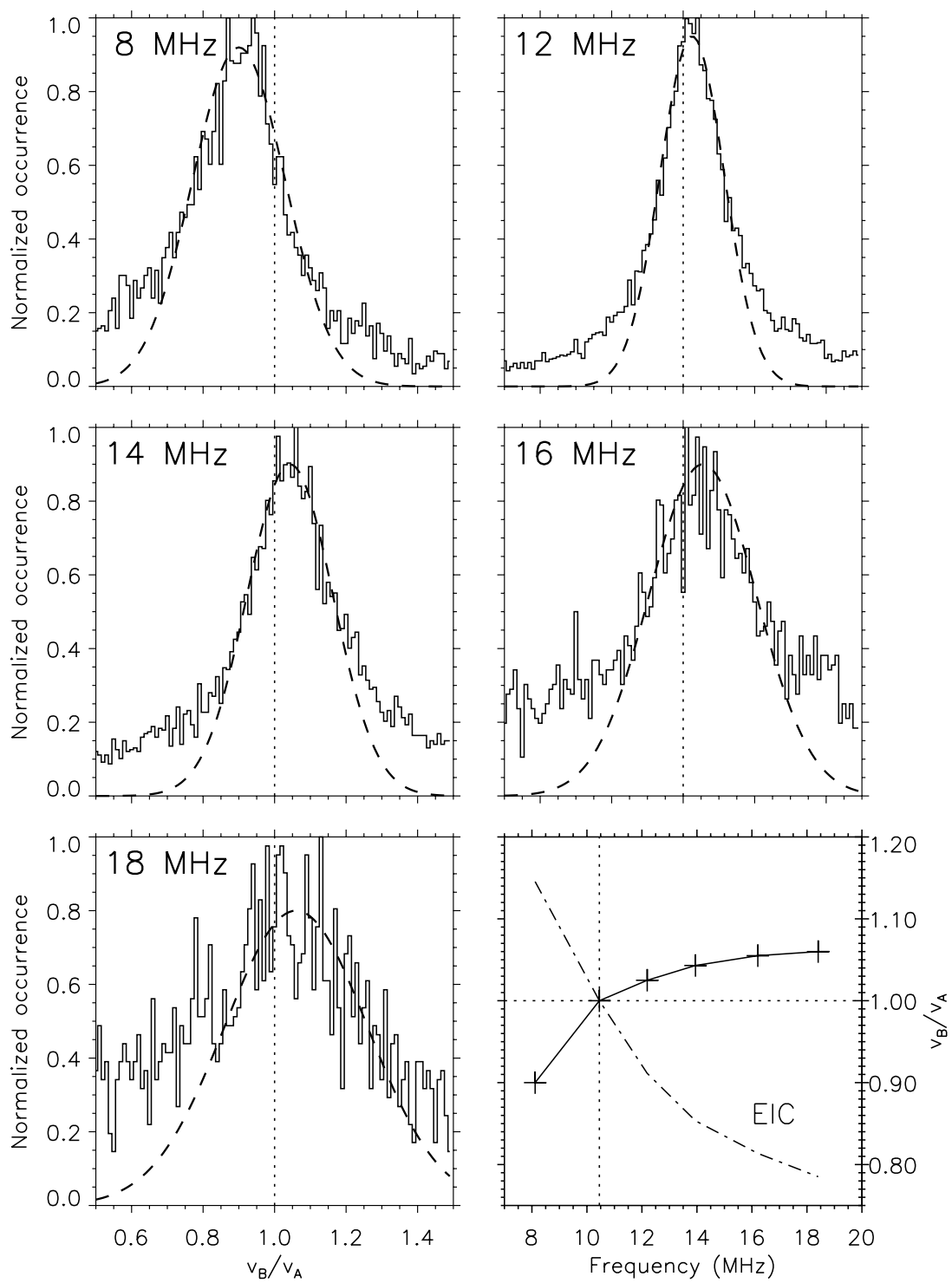

Fig. 13. Occurrence distributions of the ratio $v_{B} / v_{A}$ for high-Doppler shift echoes of Fig. 8 (region 1). Superimposed on each is a bestfit Gaussian (dashed curve). (Bottom right) The variation of $v_{B} / v_{A}$ with radar frequency (solid curve and crosses). Superimposed is the expected variation for electrostatic ion cyclotron waves (dot-dashed curve).

observed by the Finland radar at this time is scattered from the F-region, and as a consequence, provides an accurate measure of the line-of-sight component of the plasma drift velocity $E / B$. Throughout the field-of-view the Doppler shifts are consistent with westward return convection flow, as expected in the eastward electrojet region, that is plasma drift towards and away from the radar in eastward- and westwardpointing beams, respectively. Here we concentrate on the flow measured below $70^{\circ}$ latitude, at similar latitudes to the E-region backscatter observed by the Iceland radar. Figure $14 \mathrm{~b}$ shows the line-of-sight velocity for all echoes below $70^{\circ}$ latitude plotted as a function of L-shell angle. In addition, Fig. 14a shows a small patch of F-region echoes in the far ranges of the eastward-pointing beams of the Iceland radar field-of-view (purple patch). These eastward-pointing beams should measure almost the full magnitude of the re- turn flow, and indeed these echoes have Doppler shifts of the order of $1100 \mathrm{~m} \mathrm{~s}^{-1}$ (see also Fig. 14b). If the convection flow is uniform across this region, then a cosine dependence in the line-of-sight velocities is expected, as demonstrated by the fit-by-eye cosine curve (dashed line). This fit suggests a flow speed near $1100 \mathrm{~ms}^{-1}$, rotated from the L-shell direction by approximately $15^{\circ}$. Hence, we can be quite confident that the electric field in the return flow region is in excess of $50 \mathrm{mV} \mathrm{m}^{-1}$. In this case, field-aligned plasma drift is not necessary for the generation of $C_{S}$ or super- $C_{S}$ E-region echoes. Dashed curves in Fig. 12 indicate the line-of-sight component of $E / B$, demonstrating that all E-region echoes have Doppler shifts below this. This further shows that the Doppler shifts of the super- $C_{S}$ echoes appear to follow a similar cosine dependence, but are depressed below it by a few hundred $\mathrm{m} \mathrm{s}^{-1}$. 

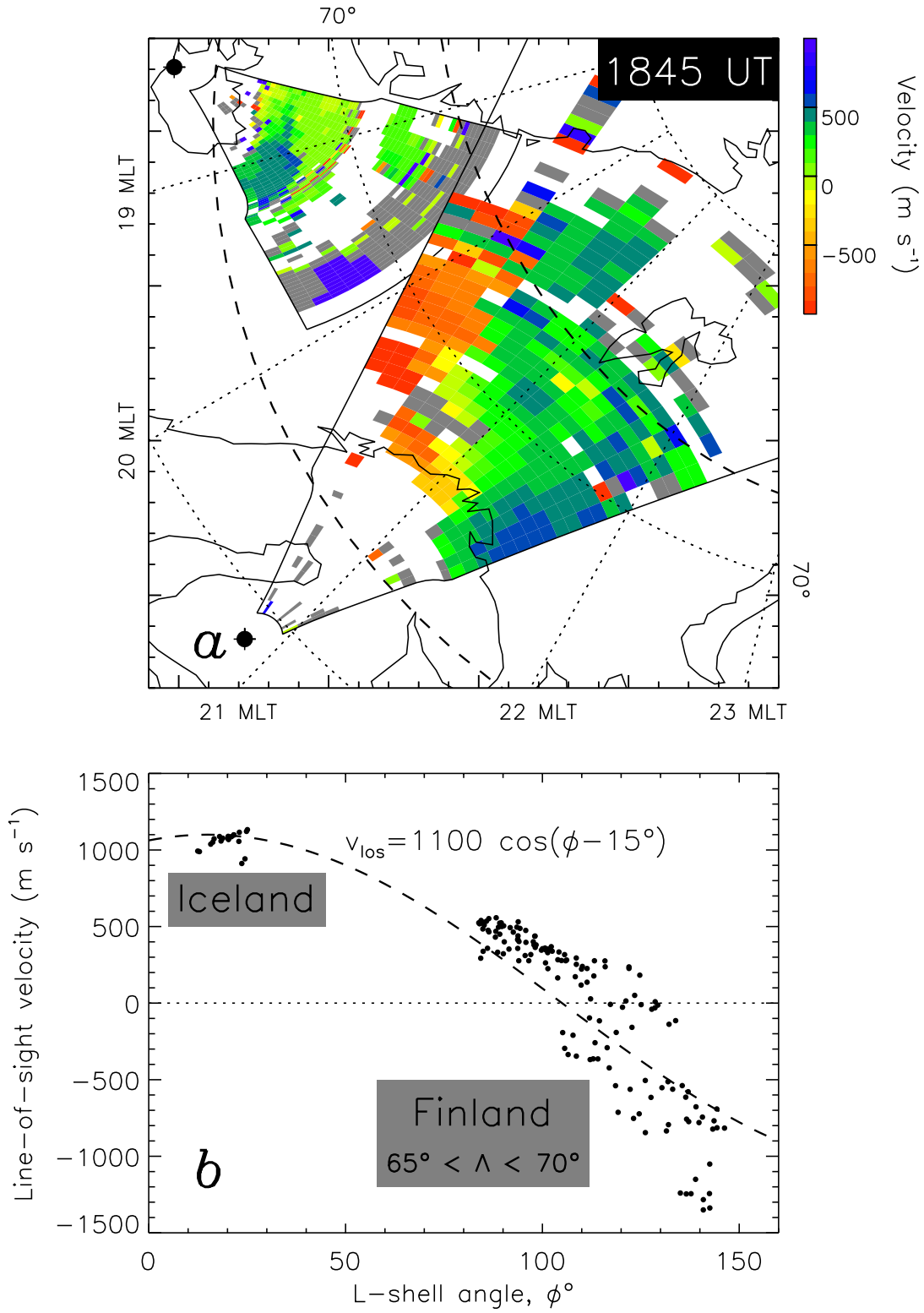

Fig. 14. (a) Magnetic latitude and MLT plot of Doppler shift observed in channel A of the Iceland East radar for the scan starting 18:44:57 UT (top left), and simultaneous observations from the Finland radar (bottom left). Dashed lines show the extent of the statistical auroral oval. (b) Doppler shift as a function of L-shell angle for the Finland and Iceland East F-region observations below $70^{\circ}$ magnetic latitude. Superimposed is a dashed curve indicating $v_{\text {los }}=1100 \cos \left(\phi-15^{\circ}\right)$.

Even so, other problems emerge with the ion cyclotron wave interpretation of the super- $C_{S}$ echoes. The velocity of these echoes shows a distinct flow angle dependence, but the dispersion relationship of ion cyclotron waves, $\omega_{r}^{2}=$ $\Omega_{i}^{2}+k^{2} C_{S}^{2}$ (where $\omega_{r}$ is the real part of the wave frequency and $\Omega_{i}$ is the gyrofrequency of the relevant ion species), suggests that their phase speed $\left(\omega_{r} / k\right)$ should be independent of look-direction. It is also not clear why the maximum in the velocity of these high Doppler shift echoes always appears at intermediate flow angles, as in the present observations and those of Villain et al. $(1987,1990)$ and Milan and Lester (2001). Furthermore, the dispersion relationship suggests that the phase velocity of the EIC waves should de- crease with increasing $k$ (see curve superimposed on Fig. 13), whereas the present observations suggest the opposite dependence.

Interpretation of these echoes in terms of gradientstabilized or gradient-destabilized two-stream waves is also problematic. Depending on the orientation of gradients in electron density with respect to the magnetic and electric fields, the threshold electric field for generation of twostream waves (and hence also their phase velocity) can be significantly enhanced or depressed (e.g. Fejer et al., 1984). Horizontal and vertical electron density gradients were implicated in this process by St.-Maurice et al. (1994) and Haldoupis et al. (2000), respectively. The latter case is espe- 
cially attractive as a strong upward vertical electron density gradient is always present at the bottom of the E-layer and a downward gradient is often present at the top of the layer (Haldoupis et al., 2000). This could automatically explain the altitude-stratification of the echoes observed. In the eastward electrojet, where the convection electric field points northwards, the gradient in the bottomside E-region should destabilize the ionosphere such that irregularities are generated at threshold velocities significantly below $C_{S}$. In the topside E-region the gradient should stabilize the ionosphere, and threshold velocities above $C_{S}$ are necessary to produce irregularities. This is perhaps consistent with the observations in the eastward electrojet on the night of 14-15 December. In the westward electrojet, where the electric field points southwards, the situation should be reversed such that the top- and bottom-sides of the E-region are destabilized and stabilized, respectively. We have no clear evidence for this reversed stratification, and previous studies have always shown high Doppler shifts to originate from higher altitudes (Milan et al., 2001). Two additional problems arise. The effect of the gradient on the instability threshold should decrease with increasing $k$ (for this reason it is not expected to be significant for VHF observations), opposite to the dependence observed. Also, the exact geometry of magnetic field, electric field, radar wave vector, and electron density gradient which gives rise to the observed flow angle dependence of Doppler shift is difficult to envisage as the de/stabilization is expected to maximize for radar look-directions along the electrojet flow, inconsistent with the observations. This has been discussed with specific attention to horizontal gradients by Milan and Lester (2001), though the argument equally applies to vertical gradients.

An alternative cause for the low Doppler shift echo population, significantly depressed below the line-of-sight component of the electron drift, is that they appear at lower altitudes than suggested by our interferometric measurements. The dispersion relationship of gradient drift waves gives $\omega_{r}=k V_{d} \cos \theta /(1+\Psi)$, where $V_{d}$ is the relative drift between electrons and ions (we assume ions are stationary), $\theta$ is the flow angle as before, and $\Psi$ depends on the ratio of the ion and electron collision and gyrofrequencies $\Psi=v_{e} v_{i} / \Omega_{e} \Omega_{i}$ (for field-aligned irregularities). $\Psi$ increases with decreasing altitude, such that in the lower Eregion, say near $90 \mathrm{~km}$ it can approach values of 10 . In this case the phase speed of the irregularities $\left(\omega_{r} / k\right)$ would be suppressed to approximately $10 \%$ of the electron drift, as observed. Further work would be required to understand why we should receive echoes so frequently from so low in the Eregion. Alternatively, $\Psi$ also increases at high aspect angles, that is for irregularities that are not exactly aligned along the magnetic field.

Finally, we note one last possible explanation for the increase in Doppler shift with radar frequency: at higher frequencies the scatter may originate from higher altitudes within the E-region, that is at altitudes where the ambient ion-acoustic speed is greater. Radar backscatter is observed where the radar beam approaches orthogonality with the magnetic field. As the magnetic field points essentially vertically at polar latitudes, the radar beam must be refracted to close to its reflection criterion at the point where scatter is detected. As discussed by Milan and Lester (2001), ground scatter is frequently observed at twice the range of E-region ionospheric, supporting this assertion. It is well known that the altitude of reflection within the ionosphere depends on radio frequency, such that higher frequencies are reflected at higher altitudes where the electron density is greater. Thus, the altitude from which backscatter is received could tend to be somewhat greater at the higher frequencies, probing altitudes where the ambient ion-acoustic speed is also higher. In this case, the trend to higher Doppler shifts at higher frequencies could be due more to propagation considerations than a $k$-dependence of the plasma instabilities.

\section{Summary and conclusions}

Multi-frequency HF radar observations of backscatter from the E-region within the auroral electrojets show that echo spectra tend to contain both a low and a high Doppler shift component, though often one or the other component dominates. We term such spectra two-peak E-region echoes or TWOPEEs. In the eastward electrojet high Doppler shift echoes appear to originate from a higher altitude than low Doppler shift echoes; the situation is less clear in the westward electrojet. At low flow angles, when the radar is pointing roughly zonally along the direction of electrojet flow, echoes are narrow and Doppler shifted to near the ionacoustic speed, the characteristics of type I waves observed by VHF radars. At greater flow angles, though still within the "cone of instability" of two-stream waves, two echo populations are seen, both broader, and with high (greater than $C_{S}$ ) and low (generally less than $200 \mathrm{~m} \mathrm{~s}^{-1}$ ) Doppler shifts. The former would be identified as type IV and the latter a mixture of types II and III in VHF nomenclature. The maximum Doppler shifts observed in electrojet backscatter, the super- $C_{S}$ echoes, do not occur when looking along the direction of electron drift, but at intermediate flow angles near $45^{\circ}$ (see also Milan and Lester, 2001). At 10 and $12 \mathrm{MHz}$ these echoes have the greatest backscatter power of all electrojet returns, though their cross section appears to fall off steeply at higher and lower frequencies. This frequency dependence argues against such echoes arising in regions where the ionacoustic speed is elevated due to heating, an effect which should not depend on the wavelength of the diagnostic radio wave. Plasma drift measurements from the Finland radar suggest that the background electric field is sufficient to account for the high Doppler shifts observed, and field-aligned electron motions are not required.

It has previously been postulated that the low Doppler shift population, as type II echoes scattered from gradient drift waves, give the line-of-sight component of the electron drift velocity or $E / B$ from which the background electric field can easily be estimated (e.g. Villain et al., 1987, 1990). However, our present observations suggest that they give a gross 
underestimate of the electric field. We speculate that these echoes may arise in the destabilizing electron density gradient present at the bottom of the E-layer in the eastward electrojet, in a manner similar to that described by Haldoupis et al. (2000), or may arise at low altitudes where $\Psi$ is large. A stabilizing gradient, giving rise to super- $C_{S}$ echoes could also be present at the top of the E-layer. It is not clear why these echoes should only appear at intermediate flow angles unless they are secondary waves of some sort. Also, the $k$-dependence of the Doppler shift of the super- $C_{S}$ echoes, an increase in velocity with increasing $k$, seems inconsistent with the expected wave characteristics.

To fully resolve this issue, co-located measurements of the electric field are necessary. We propose to employ low-Earth orbit satellites such as FAST and DMSP to provide electric field measurements over the electrojet regions and within the field-of-view of the Iceland East radar, and thus to determine the true relationship between $E$ and Doppler shift.

Acknowledgements. CUTLASS is supported by the Particle Physics and Astronomy Research Council (PPARC grant no. PPA/R/R/1997/00256), UK, the Swedish Institute for Space Physics, Uppsala, and the Finnish Meteorological Institute, Helsinki.

The Editor in Chief thanks M. Uspensky and another referee for their help in evaluating this paper.

\section{References}

Balsley, B. B. and Ecklund, W. L.: VHF power spectra of the radar aurora, J. Geophys. Res, 77, 4746-4760, 1972.

Fejer, B. G., Providakes, J., and Farley, D. T.: Theory of plasma waves in the auroral E-region, J. Geophys. Res., 89, 7487-7494, 1984.

Foster, J. C. and Erickson, P. J.: Simultaneous observations of Eregion coherent backscatter and electric field amplitude at Fregion heights with the Millstone Hill UHF radar, Geophys. Res. Lett., 27, 3177-3180, 2000.

Greenwald, R. A., Baker, K. B., Dudeney, J. R., Pinnock, M., Jones, T. B., Thomas, E. C., Villain, J.-P., Cerisier, J.-C., Senior, C., Hanuise, C., Hunsucker, R. D., Sofko, G., Koehler, J., Nielsen, E., Pellinen, R., Walker, A. D. M., Sato, N., and Yamagishi, H.: DARN/SuperDARN: A global view of the dynamics of highlatitude convection, Space Sci. Rev., 71, 761-796, 1995.

Haldoupis, C.: A review on radio studies of the auroral E-region ionospheric irregularities, Ann. Geophysicae, 7, 239-258, 1989.

Haldoupis, C., Schlegel, K., and Hussey, G.: Auroral E-region electron density gradients measured with EISCAT, Ann. Geophysicae, 18, 1172-1181, 2000.

Hall, G. E., MacDougall, J. W., Moorcroft, D. R., St.-Maurice, J.P., Manson, A. H., and Meek, C. E.: Super Dual Auroral Radar Network observations of meteor echoes, J. Geophys. Res., 102, 14 603-14 614, 1997.

Hanuise, C., Villain, J.-P., Cerisier, J.-C., Senior, C., Ruohoniemi, J. M., Greenwald, R. A., and Baker, K. B.: Statistical study of highlatitude E-region Doppler spectra obtained with the SHERPA
HF radar, Ann. Geophysicae, 9, 273-285, 1991.

Hanuise, C., Villain, J.-P., Gresillon, D., Cabrit, B., Greenwald, R. A., and Baker, K. B.: Interpretation of HF radar ionospheric Doppler spectra by collective wave scattering theory, Ann. Geophysicae, 11, 29-39, 1993.

Keys, J. G. and Johnston, P. V.: Radio aurora dynamics as seen by Doppler radar, Geophys. Res. Lett., 6, 97-100, 1979.

Koustov, A. V., Danskin, D. W., Uspensky, M. V., Ogawa, T., Janhunen, P., Nishitani, N., Nozawa, S., Lester, M., and Milan, S.: Velocities of auroral coherent echoes at 12 and $144 \mathrm{MHz}$, Ann. Geophysicae, 20, 1647-1661, 2002.

Milan, S. E., Jones, T. B., Robinson, T. R., Thomas, E. C., and Yeoman, T. K.: Interferometric evidence for the observation of ground backscatter originating behind the CUTLASS coherent HF radars, Ann. Geophysicae, 15, 29-39, 1997.

Milan, S. E. and Lester, M.: Spectral and flow angle characteristics of backscatter from decametre irregularities in the auroral electrojets, Adv. Space Res., 23, 1773-1776, 1999.

Milan, S. E. and Lester, M.: A classification of spectral populations observed in HF radar backscatter from the E-region electrojets, Ann. Geophysicae, 19, 189-204, 2001.

Milan, S. E., Lester, M., Sato, N., and Takizawa, H.: On the altitude dependence of the spectral characteristics of decametrewavelength E-region backscatter and the relationship with optical auroral forms, Ann. Geophysicae, 19, 205-217, 2001.

Milan, S. E., Sato, N., Lester, M., Yeoman, T. K., Murata, Y., Doi, H., and Saemundsson, T.: The spectral characteristics of E-region radar echoes co-located with and adjacent to visual auroral arcs, Ann. Geophysicae, 20, 795-805, 2002.

Sahr, J. D. and Fejer, G. F.: Auroral electrojet plasma irregularity theory and experiment: A critical review of present understanding and future directions, J. Geophys. Res., 101, 26 893-26909, 1996.

St.-Maurice, J.-P., Prikryl, P., Danskin, D. W., Hamza, A. M., Sofko, G. J., Koehler, J. A., Kustov, A., and Chen, J.: On the origin of narrow non-ion-acoustic coherent radar spectra in the highlatitude E-region, J. Geophys. Res., 99, 6447-6474, 1994.

Uspensky, M. V., Koustov, A. V., Eglitis, P., Huuskonen, A., Milan, S. E., Pulkkinen, T., and Pirjola, R.: CUTLASS HF radar observations of high-velocity E-region echoes, Ann. Geophysicae, 19, 411-424, 2001.

Uspensky, M. V., Kustov, A. V., Sofko, G. J., Koehler, J. A., Villain, J.-P., Hanuise, C., Ruohoniemi, J. M., and Williams, P. J. S.: Ionospheric refraction effects in slant range profiles or auroral HF coherent echoes, Radio Sci., 29, 503-517, 1994.

Villain, J.-P., Greenwald, R. A., Baker, K. B., and Ruohoniemi, J. M.: HF radar observations of E-region plasma irregularities produced by oblique electron streaming, J. Geophys. Res., 92, 12 327-12 342, 1987.

Villain, J.-P., Hanuise, C., Greenwald, R. A., Baker, K. B., and Ruohoniemi, J. M.: Obliquely propagating ion acoustic waves in the auroral E-region: Further evidence of irregularity production by field-aligned electron streaming, J. Geophys. Res., 95, 7833-7846, 1990.

Watermann, J., McNamara, A. G., Sofko, G. J., and Koehler, J. A.: Distribution of mean Doppler shift, spectral width, and skewness of coherent 50-MHz auroral radar backscatter, J. Geophys. Res., 94, 6979-6985, 1989. 